\title{
Marginal defects modified graphene with S-C-N-C groups for highly selective oxygen reduction to $\mathrm{H} 2 \mathrm{O} 2$
}

\section{Zhixing Mou}

Shanxi University

\section{Yue-Wen Mu}

Shanxi University https://orcid.org/0000-0002-0162-5091

Lijia Liu

University of Western Ontario

\section{Daili Cao}

Shanxi University

\section{Shuai Chen}

Institute of Coal Chemistry

\section{Wenjun Yan}

Institute of Coal Chemistry

\section{Haiqing Zhou}

Hunan Normal University

\section{Ting-Shan Chan}

National Synchrotron Radiation Research Center https://orcid.org/0000-0001-5220-1611

\section{Lo-Yueh Chang}

National Synchrotron Radiation Research Centre

\section{Yumin Qian}

Beijing Institute of Technology

\section{Xiujun Fan ( $\nabla$ fxiujun@gmail.com )}

Shanxi University https://orcid.org/0000-0003-4849-4305

\section{Article}

Keywords: Defect, hydrogen peroxide, electrocatalysis, graphene, metal-free

Posted Date: October 11th, 2021

DOI: https://doi.org/10.21203/rs.3.rs-912514/v1 
License: (c) (i) This work is licensed under a Creative Commons Attribution 4.0 International License. Read Full License 


\section{Marginal defects modified graphene with $\mathrm{S}-\mathrm{C}-\mathrm{N}-\mathrm{C}$ groups}

2 for highly selective oxygen reduction to $\mathrm{H}_{2} \mathrm{O}_{2}$

3 Zhixing Mou $^{1,2,8}$, Yuewen Mu ${ }^{2,8}$, Lijia Liü ${ }^{3}$, Daili Cao ${ }^{1,2}$, Shuai Chen ${ }^{4}$, Wenjun Yan ${ }^{4}$,

4 Haiqing Zhou ${ }^{5}$, Ting-Shan Chan ${ }^{6}$, Lo-Yueh Chang ${ }^{6}$, Yumin Qian ${ }^{7 *}$, and Xiujun Fan ${ }^{1,2 *}$

$5 \quad{ }^{1}$ Institute of Crystalline Materials, Shanxi University, Taiyuan, Shanxi 030006, China.

$6 \quad{ }^{2}$ Institute of Molecular Science, Shanxi University, Taiyuan, Shanxi 030006, China.

$7 \quad{ }^{3}$ Department of Chemistry, University of Western Ontario, London, Ontario, N6A 5B7

8 Canada.

$9{ }^{4}$ State Key Laboratory of Coal Conversion, Institute of Coal Chemistry, Chinese 10 Academy of Sciences, Taiyuan 030001, China.

$11{ }^{5}$ Key Laboratory of Low-Dimensional Quantum Structures and Quantum Control of 12 Ministry of Education, Department of Physics, Hunan Normal University, Changsha 13 410081, China.

$14{ }^{6}$ National Synchrotron Radiation Research Centre, 101 Hsin-Ann Road, Hsinchu, 15 30076, Taiwan.

$16 \quad{ }^{7}$ Beijing Key Lab of Nanophotonics \& Ultrafine Optoelectronic Systems, and School of 17 Physics, Beijing Institute of Technology, Haidian, Beijing 100081, China.

$18{ }^{8}$ These authors contributed equally: Zhixing Mou and Yuewen Mu.

19 Correspondence and requests for materials should be addressed to:

$20 Y$. M. Qian (yuminqian@,bit.edu.cn); X.J.Fan (fxiujun@gmail.com)

21 Abstract

22 Developing efficient metal-free catalysts to achieve electrochemical synthesis of 
hydrogen peroxide $\left(\mathrm{H}_{2} \mathrm{O}_{2}\right)$ is crucial for substituting traditional energy-intensive anthraquinone process. Heteroatom-doped carbon materials have shown great potential toward 2e-pathway for catalyzing oxygen reduction to hydrogen peroxide (ORHP). However, conventional nanocarbon electrocatalysts show slow kinetics toward ORHP due to the weak binding strength with $\mathrm{OOH}^{*}$ intermediate, resulting reduction of $\mathrm{O}_{2}$ to $\mathrm{H}_{2} \mathrm{O}$. Here, sulfur and nitrogen dual-doped graphene (SNC) electrocatalyst consisting of S-C-N-C functional group are synthesized through hydrothermal self-assembly and nitridation processes with thiourea as sulfur source. In S-C-N-C functional group, pentagon-S and pyrrolic-N are covalently grafted onto the edge of graphene and produce marginal carbon ring defects, which provide highly active sites for catalyzing ORHP. The obtained SNC catalysts deliver an outstanding ORHP activity and selectivity for $\mathrm{H}_{2} \mathrm{O}_{2}$ production, while retaining remarkable stability. The experimental and computational results reveal that marginal S-C-N-C functional groups afford an appropriate adsorption strength with $\mathrm{OOH}^{*}$ intermediate and a low reaction barrier as well, which are essential for the activity of ORHP.

\section{Keywords}

Defect, hydrogen peroxide, electrocatalysis, graphene, metal-free

\section{Introduction}

Hydrogen peroxide $\left(\mathrm{H}_{2} \mathrm{O}_{2}\right)$ is an omnipresent and extremely essential green oxidant that has been widely applied in various chemical industries and environmental remediation, especially for effluent disposal, industrial papermaking, textiles, medical industry, etc ${ }^{1}$. Based on a research report by Global Market Insight, Inc., the $\mathrm{H}_{2} \mathrm{O}_{2}$ 
market size is forecast to exceed $\$ 6.2$ billion by $2026^{2}$. Traditionally, the industrial product of $\mathrm{H}_{2} \mathrm{O}_{2}$ is mainly synthesized by anthraquinone oxidation process, which require complex technology and expensive palladium catalysts and generates an extensive volume of organic chemical wastes ${ }^{3}$. Recently, electrochemical synthesis of $\mathrm{H}_{2} \mathrm{O}_{2}$ through oxygen reduction reaction with two-electron transfer (2e-ORR) has attracted extensive attentions from both academia and industry. Compared to traditional anthraquinone process, it shows high energy efficiency, cost effectiveness, and environmental-friendly ${ }^{4}$. However, 2e-ORR process competes with the reduction of $\mathrm{O}_{2}$ to $\mathrm{H}_{2} \mathrm{O}$ via a four-electron (4e-) transfer, and thus highly requires efficient electrocatalysts that selectively promote oxygen reduction to hydrogen peroxide (ORHP). For the 2e-ORR route, noble metals and their alloy (Pd-Au, Pt-Hg, Pd-Hg, etc ${ }^{5}$.) are currently the most efficient catalysts for $\mathrm{H}_{2} \mathrm{O}_{2}$ production due to the electronic structure of Pt and Pd conformed to 2e-ORR process ${ }^{6}$. Unfortunately, the rarity and high cost of noble metals rigorously hamper their scalability. Therefore, it is highly urgent to seek active, stable, and earth-abundant alternatives to noble metal-based catalysts toward efficient electrocatalyze ORHP.

1 Owing to the low cost, controllable preparation and high conductivity, metal-free carbon materials have been intensively reported as promising candidates toward ORHP. Chiefly, oxidized carbon materials (such as oxidized carbon black ${ }^{7}$, oxidized CNTs ${ }^{8}$, oxygenated graphitic nanoplatelets ${ }^{9}$ ) have emerged as a type of promising catalyst for ORHP, due to the oxygen moieties improving the $\mathrm{OOH}^{*}$ absorption capacity of the adjacent carbon atoms. Nevertheless, oxidized carbon materials generally suffer from 
surface oxidization at high current, showing sluggish kinetics. Additionally, heteroatoms $\left(\mathrm{O}, \mathrm{N}, \mathrm{S}, \mathrm{etc}^{10,11}\right.$.) regulated carbon materials have garnered much attention, in which these heteroatoms can bond or even substitute carbon atoms with strong chemical bonding, introducing in-plane and out-of-plane defects to improve catalytic activity. Heteroatoms with highly electronegativity can incorporate into carbon lattice and enhance the distribution of $\pi$ electrons, which create a net positive charge surrounding carbon atoms, improving surface charge transfer kinetics ${ }^{12}$. Nevertheless, these anionic regulated carbon materials are commonly utilized for traditional $4 \mathrm{e}$ pathway to water because of the tendency for reducing $\mathrm{OOH}^{*}$ to $\mathrm{O}^{*}$ and $\mathrm{OH}^{*}$. More recently, several studies have manifested that defective carbon materials could catalyze ORHP, as the defects in carbon materials may act as reactive sites for oxygen adsorption or reduction during the electrocatalytic process ${ }^{13}$. Unfortunately, the extraneous topological defects are usually located in the basal plane of materials framework, which do not predominate in the catalytic process, showing remarkable limited effect on catalyzing $\mathrm{H}_{2} \mathrm{O}_{2}$. Meanwhile, the topological defects at edge afford diverse active sites for intermediate adsorption and further boost catalytic reaction as well. Erecting edged defects in nanocarbon catalysts is the crucial way to achieve both high catalytic activity and selectivity. However, practical difficulties (e.g., rigorous synthetic conditions, process complexity and difficulty, and hard configuration control) have severely limited the development of defect modified carbon catalysts through heteroatoms doping. Therefore, developing feasible heteroatoms doping schemes to construct edged defectenriched carbon structures for electrocatalytic ORHP becomes important yet 
challenging.

Herein, sulfur and nitrogen dual-doped graphene (SNC) with unique marginal defects is synthesized by hydrothermal process and controllable chemical vapor deposition (CVD) with thiourea $\left(\mathrm{CH}_{4} \mathrm{~N}_{2} \mathrm{~S}\right)$ as sulfur source. During CVD process, the inherent S-C-N configurations of thiourea are partially retained and chemically grafted onto the edge of graphene, building S-C-N-C functional group eventually, which induces numerous edged graphitic defects into SNC lattice. In S-C-N-C functional group, marginal defects consist with pentagon-S and pyrrolic-N coordinated with carbon atoms, which significantly alter surface atoms and electronic structures of graphene precursors, providing broad active edges for ORHP. The defect-enriched SNC exhibits an excellent catalytic performance that delivers over $90 \% \mathrm{H}_{2} \mathrm{O}_{2}$ selectivity at the $0.2-0.6 \mathrm{~V}$ vs. RHE (versus reversible hydrogen electrode) and $98.9 \%$ at $0.6 \mathrm{~V}$ vs. RHE, outperforming most state-of-the-art ORHP catalysts. The SNC catalyst also show excellent stability and retain $95 \%$ of initial activity after 50000 s continuous electrolysis. Density functional theory (DFT) calculations indicate that defective S-C-N-C functional groups afford desirable binding strength to $\mathrm{OOH}^{*}$ intermediates and diminish the energy barrier for $\mathrm{O}_{2}$ to $\mathrm{OOH}^{*}$, which confirms to be the active sites for ORHP. This general synthetic strategy provides a new insight to introduce marginal defects on metal-free electrocatalysts for application in advanced fields.

\section{Results}

Synthesis and characterization of SNC catalyst. The SNC electrocatalyst was synthesized through a two-step procedure, as schematically illustrated in Figure 1a. 
111 Firstly, the solution of graphene oxide (GO) and thiourea were mixed for ultrasonic 112 homogenization and then proceed hydrothermal reaction, and the pristine GO sheets 113 were reduced to reduced graphene oxide (rGO). As a result, S-doped rGO (S-rGO) was 114 obtained. Subsequently, the SNC catalyst were acquired by annealing S-rGO precursors 115 with $\mathrm{NH}_{3}$ in an $\mathrm{Ar}$ atmosphere. In addition, under the same synthesis process, $\mathrm{S}$ and $\mathrm{N}$ 116 co-doped graphene with sulfur powder as sulfur source (define as SSC) was synthesized

117 and used as a reference, together with monatomic sulfur and nitrogen doped rGO 118 (denoted as SC and NC, respectively) were also synthesized (Supplementary Table 1). 119 For comparison, a series of SNC with various CVD time and annealing temperature were also prepared (Supplementary Table 2) (see "Methods" for more details). Morphologies and structures of the SNC catalyst were characterized with scanning synthesized SNC catalyst exhibits a curly appearance and lamellar structure with abundant wrinkles and ripples (Figure 1b). The typical higher-resolution TEM (HRTEM) images elucidate that the wrinkled ultrathin flake consists of few-layered graphene with characteristic d-spacing about $0.30 \mathrm{~nm}$ (Figure 1c). As illustrated in Figure 1d, a spacing of $0.304 \mathrm{~nm}$ is further calculated with selected area electron diffraction (SAED) pattern. Notably, the interplanar spacing is much smaller than the theoretical thickness of monolayer graphene $(0.335 \mathrm{~nm})^{14}$, which can be further verified by X-ray diffraction (XRD) patterns. As illustrated in Figure 1e, an asymmetric (002) peak is exhibited along with a weak (100) peak, and these characteristics are typically shown in defect-enriched graphene. SNC and SC exhibit a higher broad peak $\left(25.7^{\circ}\right)$ 
than that of routine $\mathrm{SSC}$ and $\mathrm{NC}\left(25^{\circ}\right)$, indicating that tighter graphene layer distance

134 is actually obtained with the introduction of thiourea ${ }^{15}$. In addition, the effects of

135 annealing temperature and CVD time on the layer spacing of SNC were also studied,

136 and no significant diffraction angle shift was observed (Supplementary Figure 1).

137 Furthermore, the (002) peaks for SNC and SC sheets are more pronounced than

138 that in SSC and NC, which suggests a larger crystallite size in the $c$-axis ${ }^{16}$. It is

139 noteworthy to mention that the peak intensity of crystal surface (002) is positively

140 correlated with annealing temperature and CVD time, and this tendency arises from

141 graphitization intensified with overheating. Comparing with SSC and NC, SNC and SC

142 exhibit a distinct (004) peak, which indicates more crystal face exposure, generating

143 more active sites. This is also evident from the SEM image in Supplementary Figure 2,

144 which reveals the morphology of SSC sheets is mostly hardened block, not conducive

145 to the occurrence of electrocatalytic surface reactions. The high-angle annular dark-

146 field scanning TEM (HAADF-STEM) images clearly demonstrate the flake-like

147 structures of SNC are retained without aggregation or stacking after high-temperature

148 annealing (Figure 1f). In addition, the energy dispersive X-ray spectroscopy (EDS)

149 elemental mapping of C, N, O, and S overlap quite well, confirming both $\mathrm{N}$ and $\mathrm{S}$ have

150 been uniformly doped into SNC framework. 
a
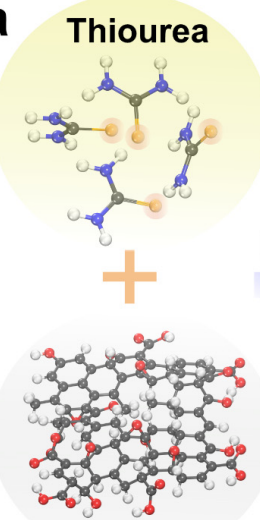

GO

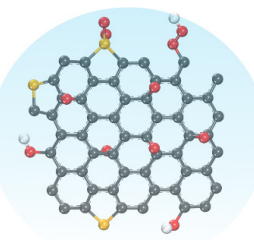

Hydrothermal
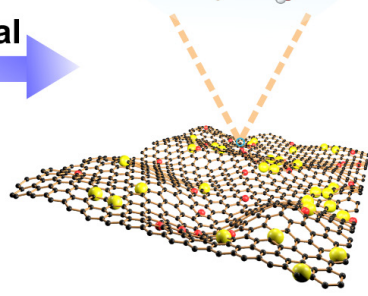

$\odot \mathrm{C}$
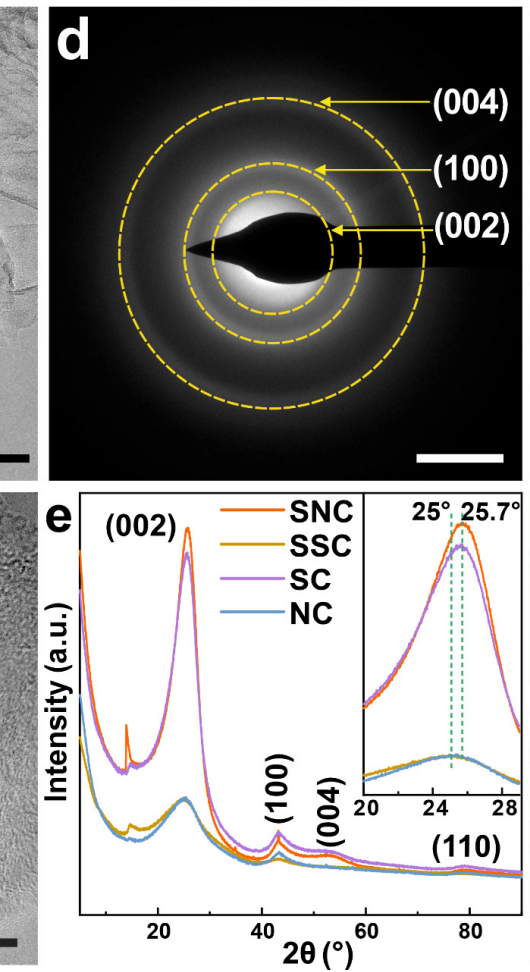

CVD

$\mathrm{NH}_{3} / \mathrm{Ar}$

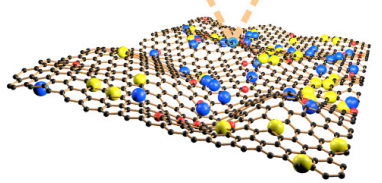

H
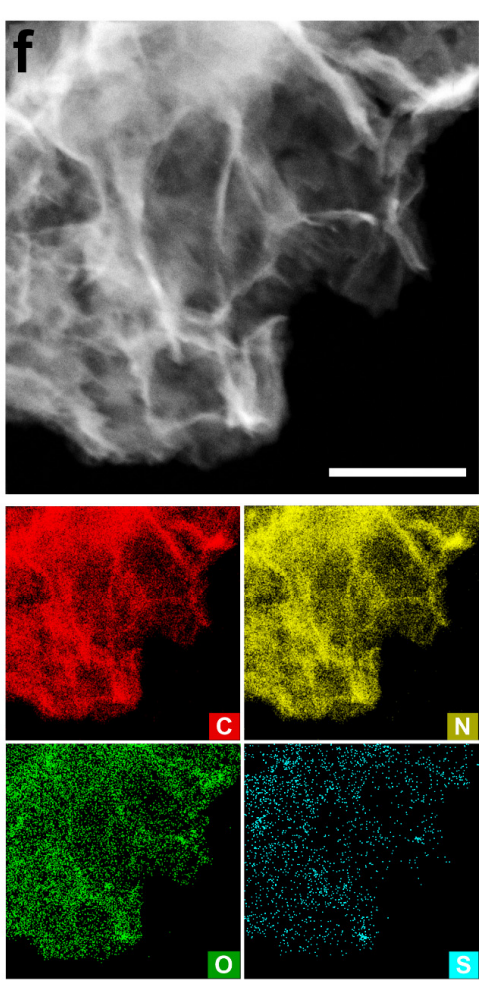

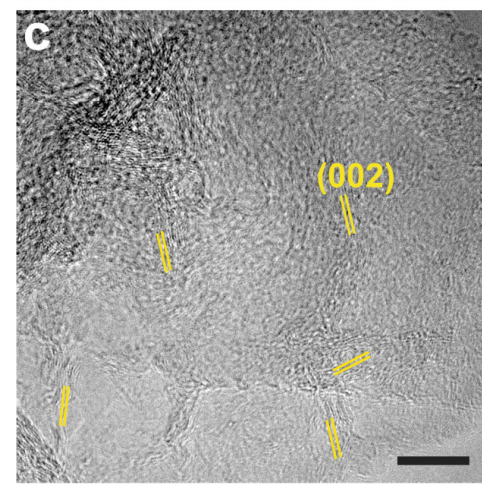

Figure 1 | Synthesis process and morphology of SNC catalyst. a, Schematic

153 illustration of the synthesis process. b, TEM image of SNC, scale bar, $100 \mathrm{~nm}$. c, HR-

TEM images of SNC with several (002) graphite surface, scale bar, $10 \mathrm{~nm}$. d,

Corresponding SAED displayed crystalline plane diffraction rings of (002), (100) and

(004), scale bar, $51 / \mathrm{nm}$. e, XRD patterns, inset is the diffraction angle migration for

SNC and SC at (002) peak. f, HAADF-STEM image and corresponding EDS elemental 
SNC moieties, Raman spectra, electron paramagnetic resonance (EPR), and X-ray photoelectron spectroscopy (XPS) were conducted. As revealed in Figure 2a, the intensity ratio of $\mathrm{D}$ and $\mathrm{G}$ band $\left(\mathrm{I}_{\mathrm{D}} / \mathrm{I}_{\mathrm{G}}\right)$ for $\mathrm{SNC}(1.147)$ is clearly higher than that of SC (1.098), NC (1.087), and SSC (1.054), suggesting that thiourea as sulfur source introduces more structural defects than that of sulfur powder. Additionally, increasing temperature could enhance the orderliness of SNC, while prolonging CVD time has the opposite effect (Supplementary Figure 3). The specific surface areas of SNC, SSC, SC and $\mathrm{NC}$ are $706,1085,579,783 \mathrm{~m}^{2} \mathrm{~g}^{-1}$, respectively (Figure $2 \mathrm{~b}$ ), and the main pore size is centered at $3.5 \mathrm{~nm}$ (Figure $2 \mathrm{~b}$, inset). Interestingly, SNC and SC have lower specific surface area than that of SSC and NC, which may attribute to the larger grain

171 size of graphene induced by thiourea, is evident in XRD patterns. In addition, the BET 172 surface area of SNC under diverse process condition were quantitatively analyzed and the SNC catalyst annealed at $850{ }^{\circ} \mathrm{C}$ for 60 min obtains maximum surface area, which 174 provides more electrocatalytic active sites for ORHP (Supplementary Figure 4). EPR 175 spectroscopy was conducted to probe unpaired electrons in graphene matrixes (Figure 176 2c). Here, a broad signal at $\mathrm{g}=2.04$ is assigned to unpaired electrons, originated from 177 nitrogen atoms, which can distribute extra electrons to adjacent carbon atoms via 178 activate $\pi$-conjugated system. While a weak symmetrical EPR signal at $\mathrm{g}=2.10$ is 179 identified as the dipole-dipole interaction, which corresponds to the inhomogeneous 180 distribution of electrons, accompanying with high electronegativity nitrogen atoms 181 bonding with carbon atoms ${ }^{17,18}$. Comparing with SSC and NC, SNC and SC modified 
with thiourea exhibit a lower EPR intensity at $\mathrm{g}=2.01$, which indicates fewer unpaired electrons are detected. Obviously, SNC and SC generate a stronger van der Waals interaction due to the decreased interplanar graphene spacing, which could accelerate electron transfer and capture more unpaired electrons.

The XPS survey spectrum shows characteristic peaks of $\mathrm{C}, \mathrm{N}, \mathrm{O}$ and $\mathrm{S}$, and corresponding atomic concentrations are obtained (Supplementary Figure 5). No evident of other impure peaks is found. As revealed in Figure 2d and Supplementary Figure 6, the $\mathrm{C} 1 \mathrm{~s}$ spectra are deconvoluted into $\mathrm{sp}^{2}$ carbon, $\mathrm{sp}^{3}$ carbon, heteroatom to carbon $(\mathrm{C}-\mathrm{O}, \mathrm{C}-\mathrm{N}, \mathrm{C}-\mathrm{S}), \mathrm{C}=\mathrm{O}, \mathrm{O}-\mathrm{C}=\mathrm{O}$ bonds at binding energies of 284.8, 285.4, 286.1, 287.0 and $289.1 \mathrm{eV}$, respectively. The $\mathrm{sp}^{3}$ carbon can be regarded as carbon atom defects, which are different from the general $\mathrm{sp}^{2}$ carbon structures, arising from the implantation of heteroatom. Apparently, SNC gives a highest $\mathrm{sp}^{3}$ carbon purity than others, highlights more extrinsic defects induced. The control samples with various annealing temperature and CVD time were also subjected to XPS measurements. For C 1s, prolonging CVD time introduces more $\mathrm{C}-\mathrm{N}$ bonds to substitute $\mathrm{C}=\mathrm{C}$ components (Supplementary Figure 7), while higher temperature accelerates graphitization and restores partial defects of $\mathrm{SNC}^{19}$ (Supplementary Figure 8). The N 1s spectra (Figure 2e) are deconvoluted into four subpeaks assignable to pyridinic-N (398.3 eV), pyrrolic-N (399.9 eV), graphitic$\mathrm{N}(401.3 \mathrm{eV})$, and N-O bonds (403.0 eV). Notably, compare to SSC and NC, SNC has a larger pyrrolic- $\mathrm{N}$ and less graphitic-N concentration, which indicates that $\mathrm{N}$ species in SNC are inclined to occur in the form of defective pentagon-C. The concentration of graphite- $\mathrm{N}$ is proportionate to the annealing temperature and CVD time; namely, 
extending nitridation time or elevating temperature can generate more graphitic-N species in the basal of carbonic ring and eliminate the pyrrolic-N (Supplementary Figure 9 and 10). Additionally, extra stretching mode of C-N heterocycles emerged with prolonging CVD time, which were detected in the Fourier-transform infrared (FTIR) spectra (Supplementary Figure 11).

As presented in Figure 2f, SNC, SSC, SC and NC exhibit $\mathrm{S}=\mathrm{O}$ peak $(534.1 \mathrm{eV})$ and oxygen-containing groups $(\mathrm{O}-\mathrm{C}=\mathrm{OH}, \mathrm{C}-\mathrm{OH}$ and $\mathrm{C}=\mathrm{O}$ at 533.4, 532.2 and $531.0 \mathrm{eV}$, respectively) after annealing, which are distributed uniformly in carbon matrix ${ }^{20}$. These results are also evidenced by the FT-IR spectra (Supplementary Figure 12). Meanwhile, compared with $\mathrm{SC}, \mathrm{SNC}, \mathrm{SSC}$, and $\mathrm{NC}$ all possess reduced $\mathrm{O}-\mathrm{C}=\mathrm{O}$ bonds accompanied with an increase in $\mathrm{C}-\mathrm{O}$ bonds, demonstrating that the carboxyl groups are partially eliminated with high-temperature nitridation process. Specially, the peak intensity of $\mathrm{C}-\mathrm{O}$ at $532.2 \mathrm{eV}$ gradually enhances with nitridation temperature increasing, which is attributed to the break of $\mathrm{C}=\mathrm{O}$ bonds in carboxyl group at high-temperature ${ }^{21}$ (Supplementary Figure 13). Similarly, most of characteristic bonds for SNC annealing at various annealing temperatures become insignificant, which are perceived in FT-IR (Supplementary Figure 14). As revealed in Figure 2g, the fitted peaks around 164.0 and $165.4 \mathrm{eV}$ are referred to the spin-orbit coupling of S $2 \mathrm{p}$ (thiophene-S and $\mathrm{C}=\mathrm{S}$ ), and sub-peaks at 168.4 and $169.6 \mathrm{eV}$ are sulfur oxide species (-C-SOx-, $\mathrm{x}=2,3)^{22}$. A small broad shoulder related with oxidized $\mathrm{S}$ is clearly observed in $\mathrm{NC}$ and no peaks are detected at the region of S $2 p$ spin-orbit, demonstrating that sulfur in SNC is effectively introduced by thiourea. Specifically, compared to SSC, the S 2p in SNC shift toward to 
higher binding energy, which can be attributed to the formation of thiophene-S, giving

227 rise to these micro-displacements ${ }^{23}$ (Supplementary Figure 15). Moreover, as

228 nitridation temperature increasing, the content of $\mathrm{O}=\mathrm{S}=\mathrm{O}$ species obviously increases

229 accompanied by the gradually decrease of $-\mathrm{SO}_{3} \mathrm{H}$ groups, indicating that the hydroxy

230 bonded coordinated with sulfur atom could be removed with high-temperature CVD

231 process (Supplementary Figure 16 and 17).
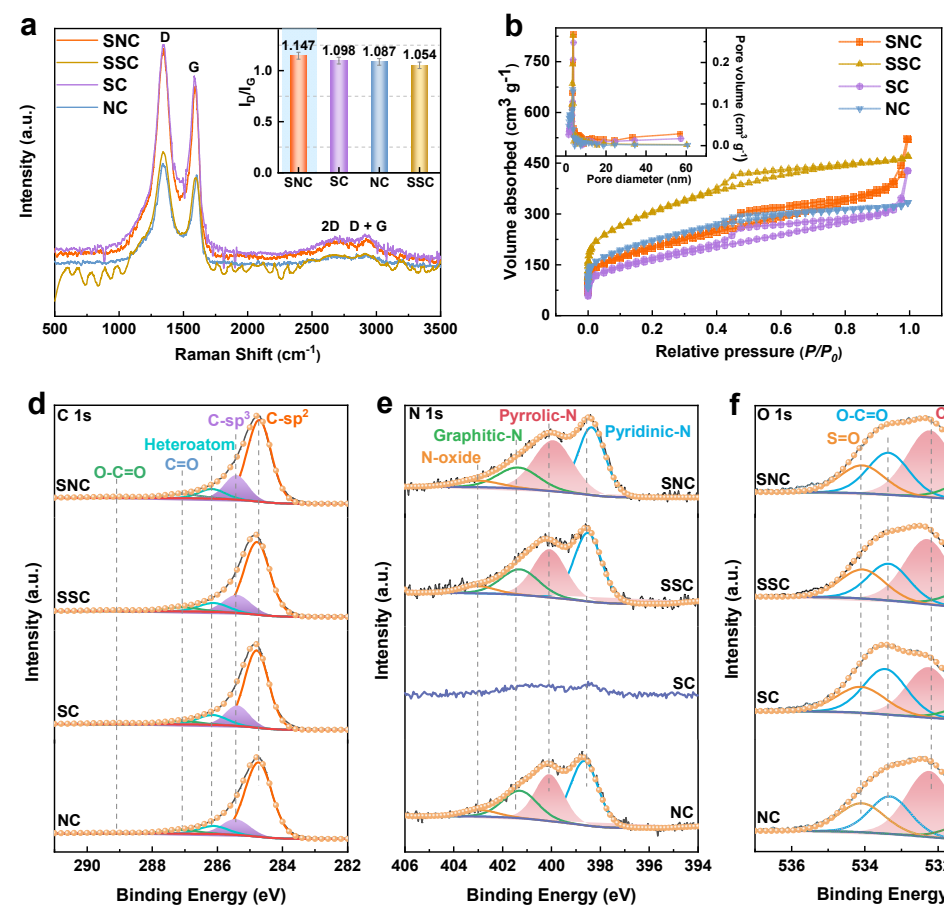
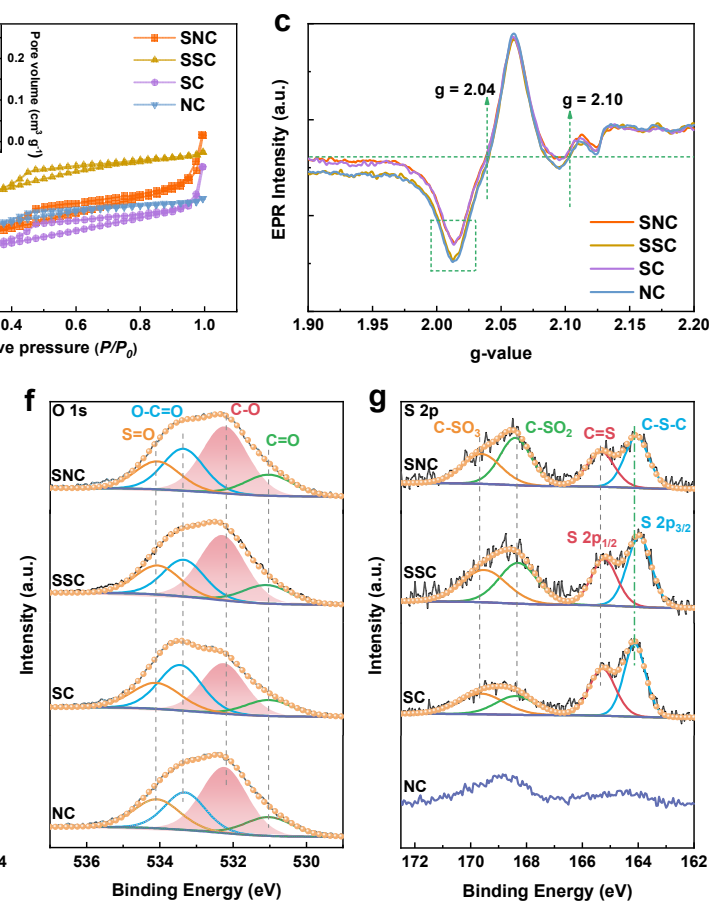

Figure 2 | Compositional characterization. a, Raman spectra of graphene matrixes, inset is the corresponding $\mathrm{I}_{\mathrm{D}} / \mathrm{I}_{\mathrm{G}}$ values. The $2 \mathrm{D}$ band is obviously enhanced in SNC and $\mathrm{SC}$, implying the number of graphene layers are decreased compared to SSC and NC. The emergence of $\mathrm{D}+\mathrm{G}$ band at $2920 \mathrm{~cm}^{-1}$ affirms the increased structure disorder. $\mathbf{b}$, Isothermal curve of $\mathrm{N}_{2}$ adsorption and desorption, inset is pore diameter distribution diagram. c, EPR spectra of graphene matrixes. d, High revolution of C 1s spectra with peaks deconvoluted into five subpeaks, while heteroatom refers to the C-S, C-N, and 
C-O bonds in graphene matrixes. e, High resolution $\mathrm{N}$ 1s spectra. The SC shows unapparent characteristic peak in $\mathrm{N} 1 \mathrm{~s}$ spectra. $\mathbf{f}$, The deconvolution of $\mathrm{O} 1 \mathrm{~s}$ spectra. $\mathbf{g}$, S 2p spectra.

Next, to further unravel coordination features of SNC moieties, soft X-ray absorption near edge structure (XANES) and solid-state nuclear magnetic resonance (ssNMR) were applied. And commercial thiourea was investigated for comparison. As illustrated in Figure 3a, the peak around $285.5 \mathrm{eV}$ is assigned to unoccupied $\pi^{*}$ states originated from the out-of-plane bonds in the $\mathrm{sp}^{2}$ bonding configuration; the signals above $292.0 \mathrm{eV}$ corresponds to excited $\sigma^{*}$ states from C-C in-plane bonds in a carbon ring structure ${ }^{9}$. Moreover, SNC and SC have an additional shoulder peak around 284 eV (left inset), which arises from vacancy defects and Stone-Wale (SW) defects induced with the incorporation of thiourea. Compared with SSC and NC, SNC and SC exhibit a weaker $\pi^{*}$ states, also reflect the rich defects ${ }^{24}$. The right inset in Figure 3a exhibits a minor resonance situated at $288.2 \mathrm{eV}$, revealing the presence of graphene interlayer states and transition to $\mathrm{sp}^{3}$ hybridized states ${ }^{25}$. In comparison to SSC and NC, SNC and corresponding to pyridinic, pyrrolic, and graphitic-N species, respectively ${ }^{27}$. The peak located at $408.6 \mathrm{eV}$ is assigned to the general transitions from $\mathrm{N} 1 \mathrm{~s}$ core level to $\mathrm{C}-\mathrm{N}$ 
with ammonia as monotonous nitrogen source shows weak $\mathrm{C}-\mathrm{N} \sigma^{*}$ states, indicating these double nitrogen sources increase the occupation of $\sigma^{*}$ states and enhance the C$\mathrm{N}$ bonding as well. Notably, raw SC without nitridation shows weak $\pi^{*}$ and $\sigma^{*}(\mathrm{C}-\mathrm{N})$ resonances in the $398-410 \mathrm{eV}$ region; these features are assigned to the nitrogen species originated from thiourea. Thus, the pyrrolic-N in SNC is partly originated from thiourea, which accelerates $2 \mathrm{e}-\mathrm{ORR}^{29}$.

S K-edge spectra (Figure 3c) indicate that SNC and SC mainly contain thiophene$\mathrm{S}(2472.8 \mathrm{eV})$ and sulfate $(2481.2,2479.2$ and $2498.3 \mathrm{eV})$ structures $^{30}$. NC displays the feature of oxidized-S, consistent with $\mathrm{S} 2 \mathrm{p}$ spectra, which is derived from the sulfate groups retained during graphene oxidation process $^{31}$. Notably, a slight deviation of photon energy between SNC and SSC is clearly observed in the inset, which contributes to the different valence states of sulfur; that is, SSC appears in the higher photon energy can be recognized to the higher electronic oxidation state of sulfur ${ }^{32}$. Compared with $\mathrm{SSC}$, the intrinsic coordination configuration (S-C-N) of thiourea produces distinctive fine structures for SNC, which cause different photon energy. In accordance with Element analysis (EA), SSC owns higher concentration of $\mathrm{O}$ and lower S than those of SNC (Supplementary Table 3), highlighting that SSC is inclined to involve more oxidized-S species. Moreover, sulfur powder could sublime at high annealing temperature, and only part of sulfur atoms can substitute carbon atoms into graphene lattice, whereas thiourea with $\mathrm{N}$ and $\mathrm{S}$ coordinated structure are more easily preserved through two-step procedure.

Furthermore, ${ }^{13} \mathrm{C}$ ssNMR was utilized to gain insights into chemical bonding of 
SNC. As revealed in Figure 3d, SNC, SC and SSC have typical signals for $\mathrm{sp}^{2}$ carbon

285 (124.3 and $125.9 \mathrm{ppm})^{33}$, whereas the resulting NC feature is not pronounced and only 286 shows a weak signal of $\mathrm{C}=\mathrm{O}$ species at $184.9 \mathrm{ppm}$, indicating that $\mathrm{S}$-doping play a vital 287 role in enhancing the graphitization. Compared with SSC, SNC has an additional sharp peak located at $128.6 \mathrm{ppm}$, which can be indexed to $\mathrm{C}=\mathrm{S}$ bonds inherited from thiourea. Besides, the peak at $137.5 \mathrm{ppm}$ is ascribed to the $-\mathrm{SO}_{3} \mathrm{H}$ groups from sulfuric acid, consistent with the results of XANES and XPS spectra. Interestingly, in SNC, SC, and SSC, plentiful oxygen-containing groups like C-O-C (56.2 ppm), O-C-O (102.1 ppm) and hydroxyl (70.5 ppm) are almost completely eliminated with annealing; only robust carboxyl survives and exhibits an obvious peak at $164.3 \mathrm{ppm}$, which endow graphene with a certain auxiliary functionalization for $2 \mathrm{e}-\mathrm{ORR}^{20}$. In the ${ }^{13} \mathrm{C}$ NMR spectroscopy of thiourea, the signal at $163.6-164.3 \mathrm{ppm}$ is S-C-N configurations resided on original framework without annealing and nitridation. Additionally, SNC and thiourea display

297 similar signals at $164.3 \mathrm{ppm}$, which are supposed to be carboxyl groups at the edge of 298 graphene. Therefore, the S-C-N configurations in thiourea are partially preserved and 299 chemically grafted onto graphene with two-step synthesis process, eventually developing into the S-C-N-C groups in SNC framework. 

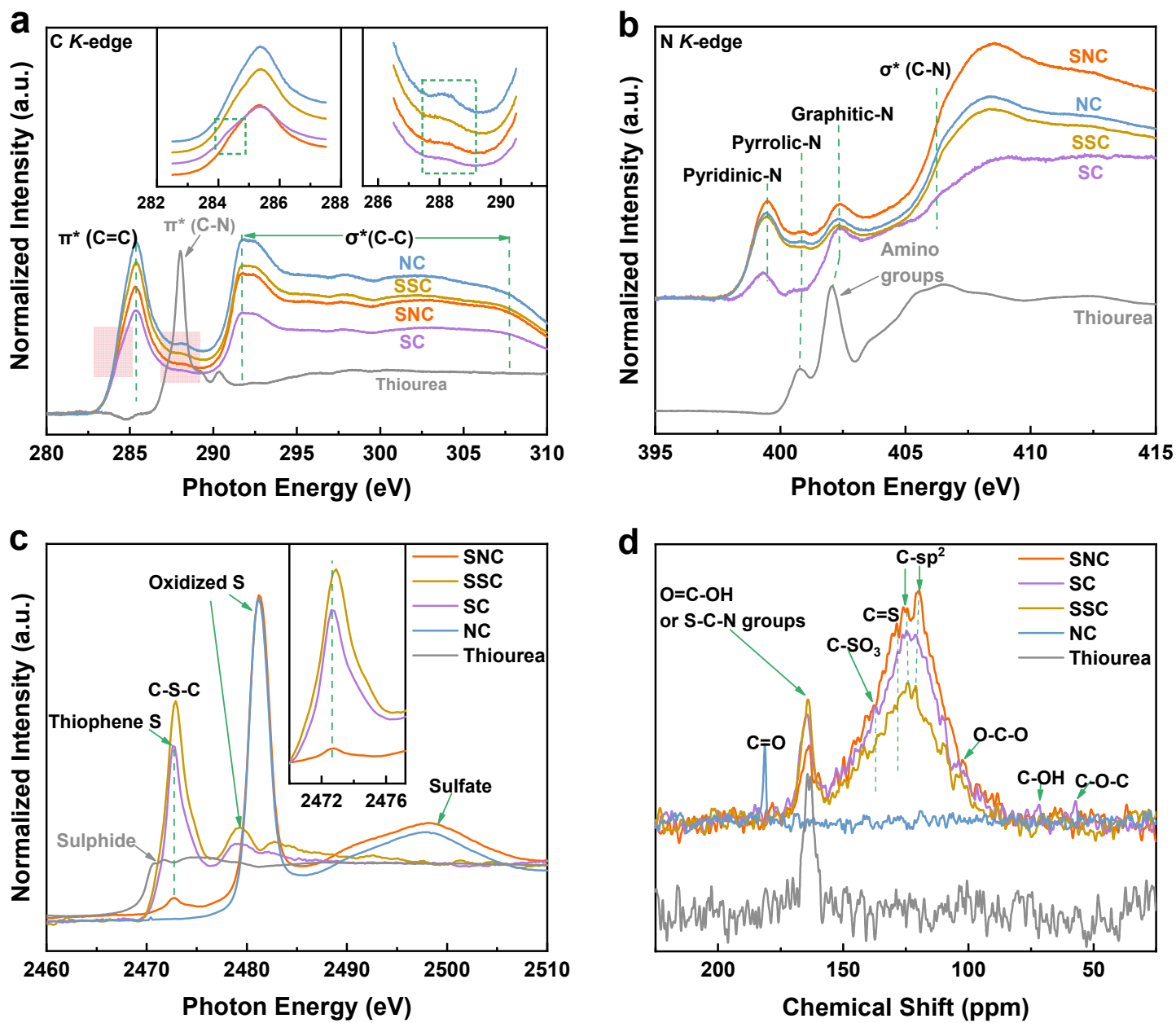

Figure 3 | Structural characterization. a, The XANES of C K-edge for graphene matrixes. Left inset is the region around $284-285 \mathrm{eV}$ and right inset is the resonances between $\pi^{*}$ and $\sigma^{*}$ states. The sharp peak at $288.0 \mathrm{eV}$ can be assigned to the $\pi^{*}$ excitation of C-N in thiourea. b, The XANES of N K-edge. The peak at 402.2 is assigned to amino groups in thiourea. c, The XANES of S-K edge, inset is the microshift for SNC and SSC. The thiourea exhibits a feeble peak of sulphide at $2470.8 \mathrm{eV}$. d, The ${ }^{13} \mathrm{C}$ NMR spectroscopy. The feature around $164.0 \mathrm{ppm}$ is assigned to S-C-N configurations from thiourea.

Microscopic morphology of SNC. Based on these results, we therefore pursued to verify the defect states of SNC via aberration-corrected scanning TEM (AC-TEM). In 
the optical images, graphene ribbon rings circled by blue hexagons are explicitly identified. These onion ring like graphene structures are composed of concentric carbon layers, and cyan pentagon signs are on behalf of disordered grain boundaries (Figure 4a). As demonstrated in Figure 4b, the graphene ribbon rings are observed again, as well as in-plane nanosized holes together with ordered graphene structures appear alternately. Remarkably, the onion ring like structures are determined by the more graphene ribbons concentrically grow on the new edges, and the high-temperature continuously ${ }^{34}$. Compared with SNC, SSC shows regular atomic configuration with few defects (Supplementary Figure 18). Thus, the S-C-N configurations of thiourea deliver a profound effect on SNC frameworks, which renders disorder and substantial atomic rearrangement in SNC. As displayed in Figure 4c, carbon vacancy and irregular aromatic rings are observed in carbon lattice of SNC. Carbon single vacancy can be ascribed Jahn-Teller distortion, which generates missing carbon atom induced by additional unsaturated dangling bond, resulting in the formation of 5-9 ring $^{35}$ (Figure $4 \mathrm{c} 1$ ). We also observe the multiple vacancies arose from a row of carbon atoms partially missing, presenting folds and bumps in SNC, which morphologically result from several dangling bonds (Supplementary Figure 19). These non-hexagonal carbon rings are actual line defect, which mostly occur in the non-sp ${ }^{2}$ hybridized orbitals, and generate 5-6-7 rings from local reconstruction (Figure 4c2 and Supplementary Figure 20). Namely, these 5-6-7 rings can account for the curvature of the SNC layers, bringing about the fluffy sheet-like structure. 

topological defects consisting of two five-atom and two seven-atom rings, through rotated a couple of $\mathrm{C}-\mathrm{C}$ bond by $90^{\circ}$ in the graphene, which can be perceived as a unique self-regulation to build stable structure of graphene lattice ${ }^{36}$. Analogously, SW defect is further depicted in Figure 4d1, which is reconstructed from four hexagonal carbon rings. Moreover, theoretical and experimental studies suggest that the carbon atoms simultaneously linked by 5-7 rings in SW defects are active sites to facilitate the ORHP ${ }^{7}$. Interestingly, various apertures of in-plane holes in SNC lattice are observed (Figure 4e and Supplementary Figure 21). These holey structures arise from the loss of numerous carbon rings caused by thermal annealing, thus forming the in-plane holes. SNC flakes obtain 55-77 rings around the in-plane holes, which are extensive enough (about $3 \mathrm{~nm}$ in diameter) and can be regarded as defective edges (Figure $4 \mathrm{e} 1$ ). Compared with the defects in basal plane, these marginal 55-77 rings are most frequently observed defects in integrated graphene, which provide broad active edges

348 for adsorption of $\mathrm{OOH}^{*}$ intermediate ${ }^{37}$. Moreover, the introduction of 55-77 rings lead the localized states transform to defect states, which open a local bandgap due to symmetry breaking and increase the density of state around the Fermi level $\left(E_{F}\right)$ as well,

351 facilitating local electron transfer ${ }^{38}$. Impressively, we find a wrinkle-rich morphology 352 with stripe phases and lattice phases in SNC, suggesting that more active sites are exposed with crumpling lattice plane (Supplementary Figure 22 and 23). Accordingly,

354 SNC catalyst with high concentration of defects are successfully synthesized, while 355 numerous marginal defects ensure great active area for electrocatalysis. 

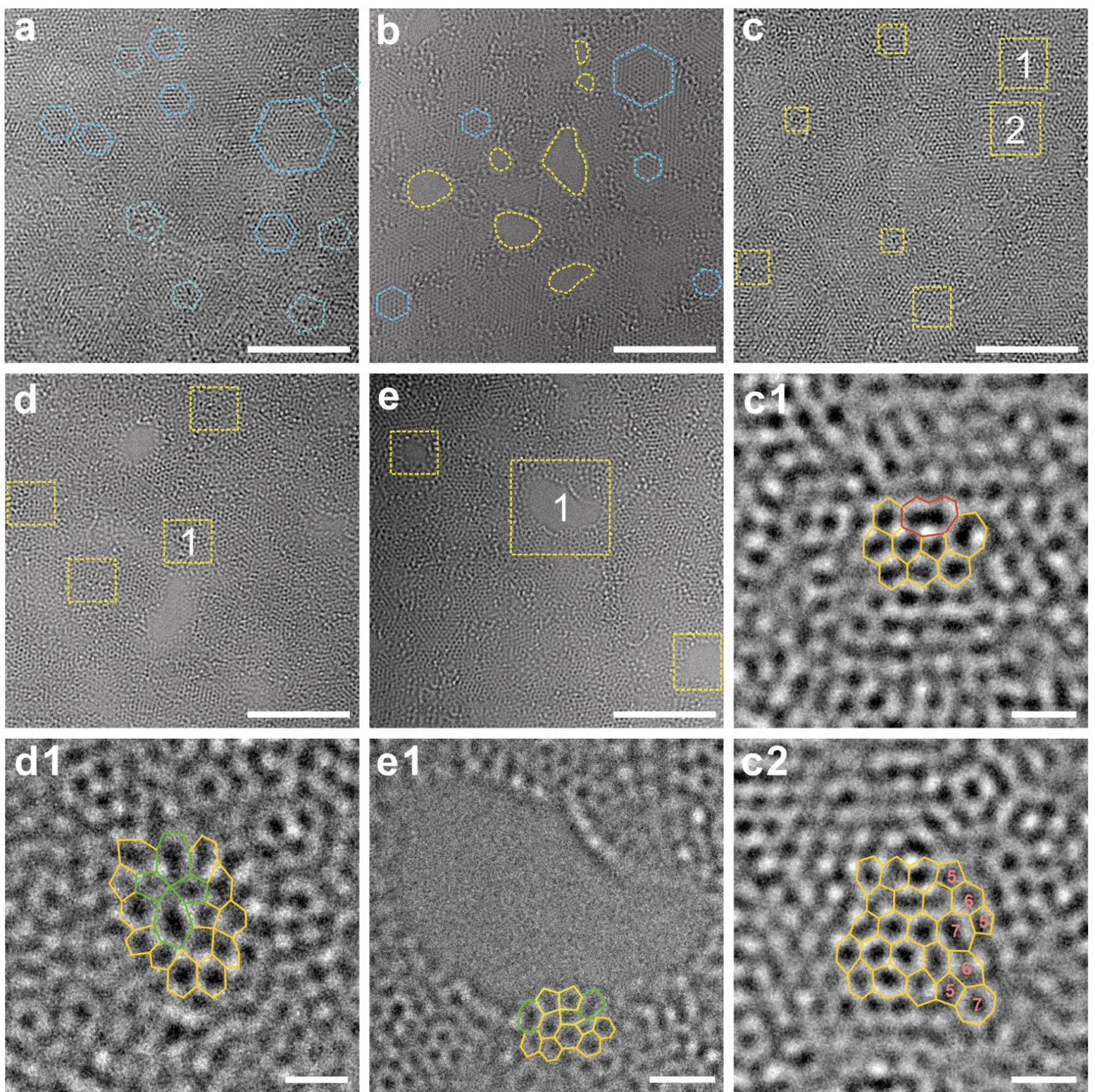

Figure 4 | AC-TEM images of SNC. a, b, The regions marked by blue and cyan

respectively correspond to regularly hexagonal onion rings and disordered grain

boundaries graphene. The region with yellow dotted line indicates that the graphene

layers are abundant of in-plane holes with size less than $5 \mathrm{~nm}$. c, c1, c2, Intrinsic defects

are revealed in the yellow region, including (c1) carbon single vacancy (c2) and non-

hexagonal rings. d, d1, Several SW defects observed in the basal plane of SNC

framework. e, e1, Holey SNC with marginal 55-77 defects at the in-plane hole. Scale

bar, $5 \mathrm{~nm}$ in a-e, $0.5 \mathrm{~nm}$ in $\mathbf{c 1 ,} \mathbf{c 2}, \mathbf{d 1}$, and e1.

Electrocatalytic ORHP characterization. To assess the ORHP activity and selectivity, 
the as-prepared SNC catalyst was directly used as working electrodes and evaluated in an $\mathrm{O}_{2}$-saturated $0.1 \mathrm{M} \mathrm{KOH}$ electrolyte by using a rotating ring-disk electrode (RRDE) device (see Method and Supplementary Figure 24 for experimental details). SSC, SC, $\mathrm{NC}$, and rGO were also measured for comparison. As displayed in linear sweep voltammetry (LSV) curves in Figure 5a, SNC renders a highest ring current density $\left(\mathrm{J}_{\mathrm{H}_{2} \mathrm{O} 2}, 0.73 \mathrm{~mA} \mathrm{~cm}{ }^{-2}\right.$ at $0.1 \mathrm{~V}$ vs. RHE) and the most positive onset potential (defined as the potential at the current density of $0.1 \mathrm{~mA} \mathrm{~cm}^{-2}$ ) of $0.8074 \mathrm{~V}$ vs. RHE, indicating the highest ORHP catalytic activity among different graphene matrixes. Nevertheless, the $\mathrm{J}_{\mathrm{H}_{2} \mathrm{O}_{2}}$ of SNC is slightly higher than that of SSC, SC, NC, and $\mathrm{rGO}(0.33,0.54,0.29$ and $0.35 \mathrm{~mA} \mathrm{~cm}^{-2}$ ), implying that the 2e pathway is preferred for SNC catalyst. For comparison, the $\mathrm{H}_{2} \mathrm{O}_{2}$ selectivity and the number of electrons transferred (n) of graphene matrixes during ORR process were also calculated. SNC renders an $\mathrm{ORR}^{29}$. Specially, with nitrogen introduction, the corresponding $\mathrm{H}_{2} \mathrm{O}_{2}$ selectivity increase from $77.8 \%$ for pure SC to $98.9 \%$ for SNC. This extreme improvement can be twice that of SSC, demonstrating the significance of thiourea as sulfur source. In detail, 
$<\mathrm{rGO}(69.6 \%)<\mathrm{SC}(77.8 \%)<\mathrm{SNC}(98.9 \%)$, while the opposite trend for $\mathbf{n}$ is observed. Surprisingly, $\mathrm{SNC}$ yields the $\mathrm{H}_{2} \mathrm{O}_{2}$ selectivity of $98.9 \%$ with the $\mathbf{n}$ value of 2.02 (Figure 5c), indicating a nearly 2e-ORR process for $\mathrm{H}_{2} \mathrm{O}_{2}$ production. Additionally, $\mathrm{rGO}$ maintains a high $\mathrm{H}_{2} \mathrm{O}_{2}$ selectivity of $\sim 70 \%$, which can be attributed to oxygen containing groups retained after annealing, accelerating 2e-ORR process ${ }^{9,20}$. Although the selectivity of rGO is insufficient as ORHP catalysts, it still affords an advantageous precursor for subsequent doping. Notably, the ORHP performance of thiourea modified graphene matrixes (SNC and SC) are superior to the counterparts without thiourea involved (SSC and NC), clearly indicating that the activity and selectivity of these catalysts are dominated by defects, which match the XANES results. Thus, the defectenriched SNC catalyst induced by thiourea can restrain 4e-ORR but expedite 2e-ORR pathway, signifying that $\mathrm{SNC}$ is the most suitable candidate for $\mathrm{H}_{2} \mathrm{O}_{2}$ production among these catalysts.

To gain more insight into kinetics of ORHP, further analysis of the Tafel slope was performed. The Tafel slope of SNC is $87 \mathrm{~mA} \mathrm{dec}^{-1}$, which is much lower than that of $\mathrm{SSC}\left(153 \mathrm{~mA} \mathrm{dec}^{-1}\right), \mathrm{SC}\left(110 \mathrm{~mA} \mathrm{dec}{ }^{-1}\right), \mathrm{NC}\left(117 \mathrm{~mA} \mathrm{dec}^{-1}\right)$, and rGO $\left(168 \mathrm{~mA} \mathrm{dec}^{-1}\right)$, signifying fast ORHP kinetics (Figure 5d). In addition, the ORHP activities of SNC prepared with various annealing temperatures and CVD time in control experiments were also evaluated (Supplementary Figure 25 and 26), and the SNC catalyst annealed at $850^{\circ} \mathrm{C}$ for $60 \mathrm{~min}$ affords faster kinetics with Tafel slope of $87 \mathrm{~mA} \mathrm{dec}^{-1}$ and higher $\mathrm{J}_{\mathrm{H}_{2} \mathrm{O} 2}$ of $0.73 \mathrm{~mA} \mathrm{~cm}{ }^{-2}$. Evidently, adjustment of temperature has a deep effect on $\mathrm{H}_{2} \mathrm{O}_{2}$ selectivity than that of CVD time (Supplementary Figure 27 and 28). Prolonging 
nitridation times and increasing annealing temperature could engender the presence graphitic-N with high concentration, thereby leading to an apparent 4e-pathway on the adjacent carbon atoms. Particularly, the increase of graphitic-N proportion with CVD time prolonging is essentially arising from the nitrogen accumulation, while graphitic$\mathrm{N}$ concentration with temperature increasing due to pyrrolic-N converted to graphitic$\mathrm{N}$ species at high-temperature. Thus, prolonging CVD time and raising annealing temperature can increase the subjects of graphitic-N in SNC, which boost 4e-ORR rather than ORHP process.

In addition, the electrochemical double-layer capacitance $\left(\mathrm{C}_{\mathrm{dl}}\right)$ measured via cyclic voltammetry $(\mathrm{CV})$ at different scan rates was probed to estimate the electrochemical surface area (ECSA) and the corresponding intrinsic activity (Figure 5e). The $\mathrm{C}_{\mathrm{dl}}$ and ECSA values of SNC are $1.78 \mathrm{mF} \mathrm{cm} \mathrm{cm}^{-2}$ and $44.5 \mathrm{~cm}^{2}$, which are higher than those of $\operatorname{SSC}\left(0.82 \mathrm{mF} \mathrm{cm}{ }^{-2}, 20.5 \mathrm{~cm}^{2}\right), \mathrm{SC}\left(0.92 \mathrm{mF} \mathrm{cm}{ }^{-2}, 23 \mathrm{~cm}^{2}\right), \mathrm{NC}\left(0.78 \mathrm{mF} \mathrm{cm}{ }^{-2}, 19.5\right.$ $\left.\mathrm{cm}^{2}\right)$, and $\mathrm{rGO}\left(0.64 \mathrm{mF} \mathrm{cm} \mathrm{cm}^{-2}, 16 \mathrm{~cm}^{2}\right)$ in $0.1 \mathrm{M} \mathrm{KOH}$ electrolyte, respectively (Supplementary Figure 29), suggesting the defect-enriched SNC systems ensure more electrocatalytically active sites exposed. Given the high ORHP activity and selectivity of SNC catalyst, we further analyzed their stability and durability to continuously catalyze the generation of $\mathrm{H}_{2} \mathrm{O}_{2}$. As presented in Figure 5f, the LSV curves of SNC in $0.1 \mathrm{M} \mathrm{KOH}$ after 10000 cycles deliver a small decrease from $\sim 2.42$ to $\sim 2.36 \mathrm{~mA} \mathrm{~cm}^{-2}$ at $0.1 \mathrm{~V}$ vs. RHE; meanwhile, the average $\mathrm{H}_{2} \mathrm{O}_{2}$ selectivity (between 0.2 and $0.6 \mathrm{~V}$ vs. RHE) is almost unchanged just with a small decline from $93.4 \%$ to $92.1 \%$, confirming the good durability. Besides, the stability of SNC catalyst was evaluated by long-term 
testing. As presented in Figure 5g, SNC catalyst retains stable ring and disk current densities over $50000 \mathrm{~s}$ of continuous operation, demonstrating a superior recyclability and stability. Excellent structural integrity of SNC is confirmed by HR-TEM images taken after the stability test, and SNC still holds a fluffy sheet-like structure without obviously morphology change, which further confirms the robustness of the SNC catalyst (Supplementary Figure 30). Specifically, Figure 5h shows a comparison map of ORHP activity for SNC against other common catalysts, including metal-oriented systems and conventional carbon nanomaterials; SNC here demonstrates highest selectivity of $98.9 \%$ at $0.6 \mathrm{~V}$ vs. RHE, which outperforms most reported electrocatalysts working in alkaline media.
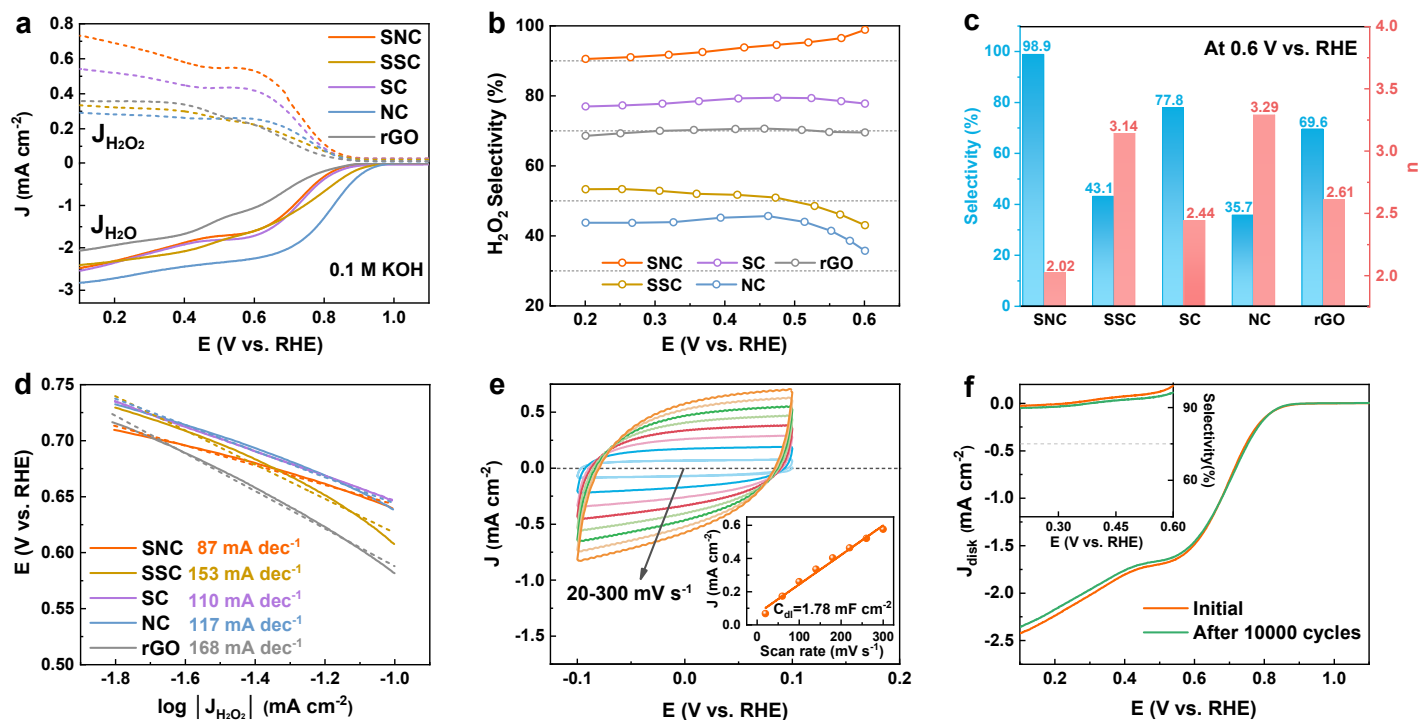

g
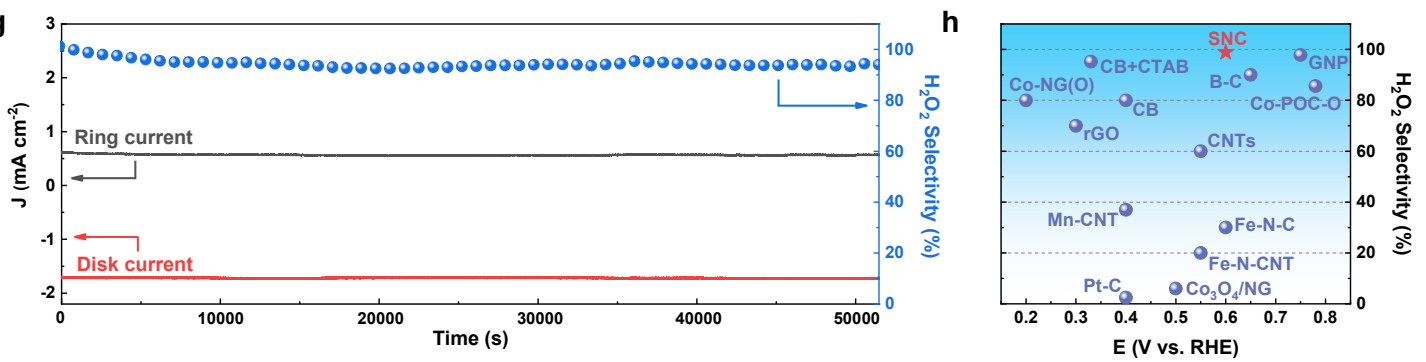

Figure 5 | ORHP performance. a, polarization curves at 1,600 rpm together with the 
corresponding $\mathrm{H}_{2} \mathrm{O}_{2}$ current on the ring electrode (dashed lines) and $\mathrm{H}_{2} \mathrm{O}$ current on the disk electrode (solid lines) for graphene matrixes. The applied potential of ring electrode was set at $1.2 \mathrm{~V}$ vs. RHE. $\mathbf{b}$, The corresponding $\mathrm{H}_{2} \mathrm{O}_{2}$ selectivity profiles at 0.2-0.6 V vs. RHE. $\mathbf{c}, \mathrm{H}_{2} \mathrm{O}_{2}$ selectivity and $\mathbf{n}$ for different systems at $0.6 \mathrm{~V}$ vs. RHE. d, The corresponding Tafel plot of kinetic current of $\mathrm{H}_{2} \mathrm{O}_{2}$. e, $\mathrm{CV}$ cycles for SNC with varying scan rates from $20-300 \mathrm{mV} \mathrm{s}^{-1}$. The inset is $\mathrm{C}_{\mathrm{dl}}$ measurement in $0.1 \mathrm{M} \mathrm{KOH}$. f, The polarization curves of SNC before and after $10000 \mathrm{CV}$ potential cycles and inset is the $\mathrm{H}_{2} \mathrm{O}_{2}$ selectivity of SNC catalyst. g, Stability measurement of SNC at a fixed disk potential of $0.40 \mathrm{~V}$ vs. RHE. h, Comparison diagram of the selectivity for $\mathrm{H}_{2} \mathrm{O}_{2}$ electrosynthesis on SNC and other reported electrocatalysts in $0.1 \mathrm{M} \mathrm{KOH}$ electrolyte.

Theoretical calculation. The above experiments demonstrate that marginal defects modified SNC with S-C-N-C groups appreciably boost ORHP. DFT simulations were then conducted to understand the catalytic mechanism of the S-C-N-C functional groups toward ORR to $\mathrm{H}_{2} \mathrm{O}_{2}$ production. Two-dimensional graphene sheet was used as model system, where four types of SNC computational models were constructed, including pentagon-S, pyrrolic-N, and hexagon-S introduced at the edge planes of the graphene (Figure 6a). Initially, we tested the doping energy of all the possible N-doped sites nearby pentagon-S, and found A position has the relatively lowest doping energy, which is the most stable SNC models (Supplementary Figure 31 and Table 5). Nevertheless, not all models create adsorption sites for $\mathrm{OOH}^{*}$. Accordingly, the adsorption energy of all carbon atoms (A-H sites) around S-C-N-C groups were calculated, in which A and B position were the most stable sites for SNC models due 
to lowest $\mathrm{OOH}^{*}$ adsorption energy (Supplementary Table 6). According to the Sabatier principle ${ }^{39}$, the critical factors for $\mathrm{O}_{2}$ conversion into $\mathrm{H}_{2} \mathrm{O}_{2}$ are the bind energy of active sites with $\mathrm{OOH}^{*}$ intermediates (equation (1)) and desorption capacity of $\mathrm{O}_{2}$ hydrogenated to $\mathrm{OOH}^{*}$ (equation (2)).

$$
\begin{gathered}
\mathrm{O}_{2}+\mathrm{H}_{2} \mathrm{O}+\mathrm{e}^{-} \rightarrow \mathrm{OOH}^{*}+\mathrm{OH}^{-} \\
\mathrm{OOH}^{*}+\mathrm{e}^{-} \rightarrow \mathrm{HO}_{2}^{-}
\end{gathered}
$$

Therefore, we used $\Delta \mathrm{GoOH}^{*}$ as the best descriptor to determine the ORHP activity on SNC. Figure $6 \mathrm{~b}$ presents the volcano plots of the $\mathrm{H}_{2} \mathrm{O}_{2}$ formation with the limiting potential $\left(\mathrm{U}_{\mathrm{L}}\right)$ of $0.70 \mathrm{~V}$, which provides a general mechanism understanding at a wide $\mathrm{pH}$ range. Evidently, the $\mathrm{UL}_{\mathrm{L}}$ closer to $0.7 \mathrm{~V}$ reflects that the selected site of SNC is more likely to catalyze ORHP, and the corresponding free energy profiles of 2e-ORR affords lower overpotential for $\mathrm{OOH}^{*}$ adsorption. Notably, most of the plots concentrate on the right side of volcano map, revealing that the binding capacity to $\mathrm{OOH}^{*}$ of active sites eventually determine the ORHP performance. Among various possible configurations for SNC groups, Edge 2A exhibits a $\mathrm{UL}_{\mathrm{L}}$ of $0.64 \mathrm{~V}$ and improves the $\mathrm{H}_{2} \mathrm{O}_{2}$ activity towards the top of the volcano plot, comparable with the previously reported noble metal catalysts ${ }^{3,5}$. Thus, we modeled Edge 4 (transforming the pentagon-S to hexagon$\mathrm{S}$ in Edge 2) to examine the effect of $\mathrm{OOH}^{*}$ adsorption by change the local electrons around sulfur atom. This conversion leads to the carbon ring doped with both nitrogen and sulfur atoms offsets z-axis from graphene base plane (Supplementary Figure 32), which induces the carbon active site become unstable, showing a lower $U_{L}(0.53 \mathrm{~V})$ and higher overpotential $(0.17 \mathrm{eV})$ for $\mathrm{OOH}^{*}$ adsorption (Figure $\left.6 \mathrm{c}\right)$. 
$0.06 \mathrm{eV}$, suggesting the optimal electronic structure. Accordingly, the free-energy diagram of the intermediate states in 4e-pathway for different SNC models are also calculated, and the energy barrier for the formation of $\mathrm{OOH}^{*}$ in $4 \mathrm{e}-$ pathway shows a similar trend with 2e-pathway (Supplementary Figure 33). We find that only Edge 1 located at the left leg of the volcano plot, which is limited by equation (2) due to the strong $\mathrm{OOH}^{*}$ binding strength, thus generating a higher dissociation energy $(3.13 \mathrm{eV})$ from $\mathrm{OOH}^{*}$ to $\mathrm{O}^{*}$ than other models, such as Edge $2(1.16 \mathrm{eV})$, Edge 3 (2.06), and Edge $4(1.77 \mathrm{eV})$. Furthermore, Edge 1A shows the highest energy barriers for $\mathrm{O}_{2}$ to $\mathrm{OOH}^{*}$ in both $2 \mathrm{e}$ - and $4 \mathrm{e}$-pathway $(0.25$ and $0.98 \mathrm{eV}$, respectively), which lead to the $\mathrm{O}-\mathrm{O}$ dissociation occurs during $\mathrm{O}_{2}$ reduction, driving the $4 \mathrm{e}$ - pathway with $\mathrm{H}_{2} \mathrm{O}$ as the major product.

To investigate the effect of oxidized-S species on the ORHP performance, we respectively replaced the positions of sulfur atoms in Edge 1 to 4 with $\mathrm{C}_{-} \mathrm{SO}_{2}$ and $\mathrm{C}$ $\mathrm{SO}_{3}$ groups, and calculated the corresponding volcano plot at same sites (Supplementary Figure 34, 35). Obviously, for $\mathrm{C}-\mathrm{SO}_{2}$ and $\mathrm{C}-\mathrm{SO}_{3}$ groups modified $\mathrm{SNC}$ models, the $\mathrm{U}_{\mathrm{L}}$ shows a gradual decrease as more oxygen atoms coordinated with pentagon-S, thereby exhibiting degraded ORHP activity (Supplementary Table 7). In addition, the introduction of oxygen atoms increases the energy of the bara configuration, leading to an unstable system with $\mathrm{OOH}^{*}$ adsorption. Particularly, only Edge $2 \mathrm{~A}$ exhibits undiminished $\mathrm{U}_{\mathrm{L}}$ and preferable $\Delta \mathrm{GoOH}^{*}$ after adding oxygen atoms, revealing the good intrinsic activity. According to previous work ${ }^{22}$, O modified S-defect 
sites have lower energy barrier for $\mathrm{OOH}^{*}$ dissociate to $\mathrm{O} *$ than that of normal S-defect sites. Thus, the $\mathrm{C}-\mathrm{SO}_{2}$ and $\mathrm{C}-\mathrm{SO}_{3}$ groups in $\mathrm{SNC}$ is more beneficial to 4e-ORR.

To verify the pyrrolic-N is favorable for $\mathrm{H}_{2} \mathrm{O}_{2}$ production in $\mathrm{S}-\mathrm{C}-\mathrm{N}-\mathrm{C}$ groups, we transformed pyrrolic- $\mathrm{N}$ to pyridinic- $\mathrm{N}$ configuration in Edge 1 and investigate the corresponding change of $\Delta \mathrm{GoOH}^{*}$ (Supplementary Figure 36). Of note, the Edge 1 doped with pyridinic-N transfers from the strongly bind side to weakly bind side, while the $\mathrm{U}_{\mathrm{L}}$ decreases from 0.45 to $0.33 \mathrm{~V}$, generating a relatively unstable system for $\mathrm{OOH}^{*}$ adsorption. This is mainly due to the delocalized lone pair electrons carried by pyridinic-N, which motivate charge jump from $\pi$ orbitals to antibonding orbitals during $\mathrm{O}_{2}$ activation, and extensively weaken the $\mathrm{O}-\mathrm{O}$ bonds as well as further facilitate $\mathrm{OOH}^{*}$ intermediate dissociation into $\mathrm{O}^{*}$ and $\mathrm{OH}^{*}$, impeding $\mathrm{ORHP}$ process ${ }^{40}$. In addition, we respectively modified the graphene model with hexagon- $\mathrm{SO}_{2}$, pentagon- $\mathrm{SO}_{2}$, pyridinic$\mathrm{N}$, pyrrolic-N, graphitic-N, pentagon-S, hexagon-S, and SW defect, and investigated the corresponding the ORHP activity of these graphene matrixes (Supplementary Figure 37). Correspondingly, for pyrrolic-N and pentagon-S modified configurations, the calculated $\mathrm{U}_{\mathrm{L}}$ is out the range of volcano plot, while pyridinic-N, graphitic-N, hexagon-S, hexagon- $\mathrm{SO}_{2}$, and pentagon- $\mathrm{SO}_{2}$ modified graphene have moderate binding energies for $\mathrm{OOH}^{*}$, which directly proves that SNC has better ORHP activity than SSC and NC (Supplementary Figure 38). Interestingly, only pyrrolic-N or pentagon-S modified graphene are limited in optimizing ORHP reaction kinetics, demonstrating the necessity of binary configurations. Thus, the pyrrolic-N and pentagon-S holistically coordinated structure in SNC framework can synergistically renders excellent 2e-ORR 
activities.

To gain more intuitive insight into the bonding nature of the SNC catalyst adsorbed with $\mathrm{O}_{2}$ molecule, we employed Crystal orbital Hamilton population (COHP) to analyze the bonding and anti-bonding states between $\mathrm{OOH}^{*}$ intermediate and active sites. As displayed in Figure 6d, Edge 1A, 3A, and 4A have several distinct bandgaps near the $E_{F}$ and respectively exhibit high overpotential of $0.25,0.10$, and $0.17 \mathrm{eV}$ (Figure 6c), which are not favorable for ORHP. Edge 2A shows a wide energy band passing through the Fermi surface, indicating that more electron transfer occurs during active sites bonding with $\mathrm{OOH}^{*}$. To give a more quantitative explanation, the integrated COHP (ICOHP) was calculated by integrating the energy up to EF. Specifically, Edge 1A has a highest ICOHP value (11.77), which contributes Edge 1A located at the left leg of the volcano plot individually; and the desorption of $\mathrm{OOH}^{*}$ limited the binding strength, leading to the strongest bonding state. Interestingly, ICOHP positively correlate with $\Delta \mathrm{GoOH}^{*}$ value, whereas Edge $2 \mathrm{~A}$ deviates from this relationship and approaches the critical value $(4.22 \mathrm{eV})$ of the ideal ORHP catalyst (Supplementary Figure 39). This deviation for Edge $2 \mathrm{~A}$ attributes to the existence of bonding states occupied by electrons in $\mathrm{EF}_{\mathrm{F}}$, which provides the least antibonding contribution with optimized binding strength toward $\mathrm{OOH}^{*}$, accounting for the best ORHP activity. Moreover, we calculated Bader charge to analyze the charge transfer on active sites before and after the adsorption of $\mathrm{OOH}^{*}$ intermediate. Compared with the sulfur and carbon atoms, the higher electronegativity of nitrogen atom $(3.04 \mathrm{eV})$ could activate $\pi$ conjugated system and have a strong ability to withdraw electron, resulting in high local 
charge density (Figure 6e, red triangle regions). Therefore, the electrons of Edge 3A adjacent to nitrogen atom are easily to be withdrawal, which produces a highest charge transfer $(0.389|\mathrm{e}|)$ than other active sites. Edge $1 \mathrm{~A}$ is the only site showing a negative Bader charge $(-0.173|\mathrm{e}|)$, which indicates the corresponding carbon atom has excess electrons outside its nucleus and strongly binds with electronegative $\mathrm{OOH}^{*}$, consisting with the results of volcano plots and COHP. As anticipated, as pentagon-S replaced by hexagon-S, the corresponding charge transfer decreases from $0.139|\mathrm{e}|$ to $0.010|\mathrm{e}|$, demonstrating the weak interaction between $\mathrm{O}_{2}$ molecules and Edge 4A, which restrains the formation of $\mathrm{OOH}^{*}$ intermediates. To further clarify the correlation between Bader charge and ORHP performance, we calculated the charge transfer of all the active sites with $\mathrm{OOH}^{*}$ adsorption (Supplementary Table 8). Compared with bara sites, most of sites absorbed with $\mathrm{OOH}^{*}$ have a higher charge transfer, only the sites of $1 \mathrm{~B}$ and $4 \mathrm{~B}$ show a subtle change $(0.438|\mathrm{e}|$ to $0.456 \mathrm{e} \mid$ and $0.294 \mathrm{e} \mid$ to $0.333 \mathrm{e} \mid$, respectively). Moreover, sites $1 \mathrm{~B}$ and 4B also exhibit low limiting potential ( 0.30 and $0.25 \mathrm{~V})$ and high overpotential values for $\mathrm{OOH}^{*}$ adsorption $(0.40$ and $0.45 \mathrm{eV}$ ) (Figure $6 \mathrm{~b}$ and $6 \mathrm{c})$. Therefore, the charge transfer for site $1 \mathrm{~B}$ and $4 \mathrm{~B}$ change insignificantly after adsorption due to weak adsorption with $\mathrm{OOH}^{*}$. According to electrostatic interaction ${ }^{41}$, the binding strength between less positively charged carbon atoms and $\mathrm{OOH}^{*}$ can be weakened, while Edge $2 \mathrm{~A}$ adsorbed with $\mathrm{OOH}^{*}$ shows a lowest charge transfer $(0.41|\mathrm{e}|)$ than other SNC models, enhancing the desorption of $\mathrm{OOH}^{*}$. 

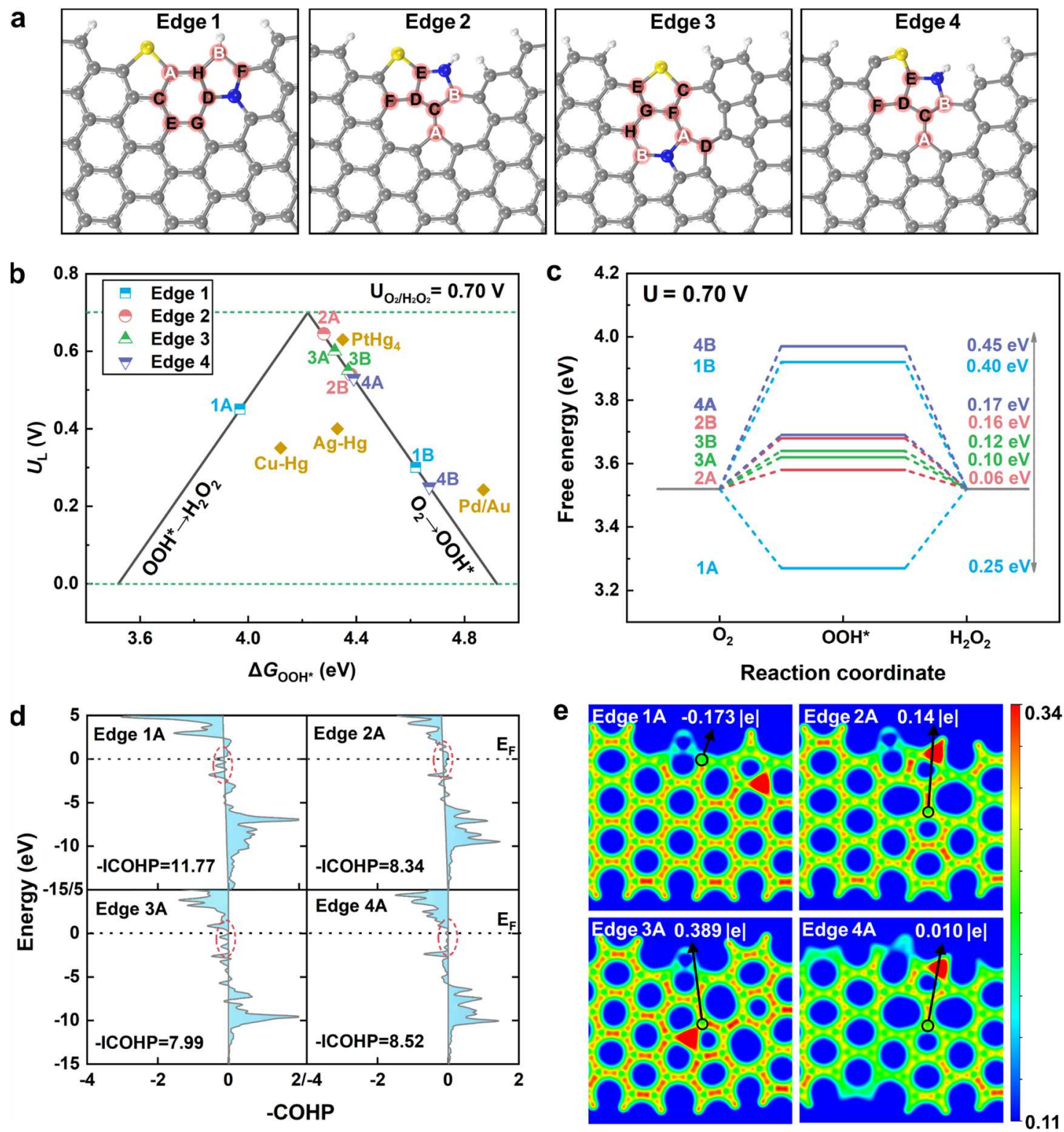

Figure 6 | Theoretical analysis of different SNC configurations. a, The atomic

577 structures of the examined SNC computational models. Gray, blue, yellow, and white

578 balls denote carbon, nitrogen, oxygen, and hydrogen atoms, respectively. The carbon

579 active sites labeled by the letters, in which A and B own the highest adsorption energies

580 for $\mathrm{OOH}^{*} . \mathbf{b}$, Calculated volcano plot (solid line) for ORHP demonstrated with limiting

581 potential $\mathrm{U}_{\mathrm{L}}$ as a function of $\Delta \mathrm{GoOH}^{*}$. The equilibrium potential for ORHP is $0.70 \mathrm{~V}$

582 and shown as the dashed green line. The golden squares present the activities of $\mathrm{Pt}-\mathrm{Hg}$,

$583 \mathrm{Cu}-\mathrm{Hg}, \mathrm{Ag}-\mathrm{Hg}$, and $\mathrm{Pd}-\mathrm{Au}$ alloys. c, Free energy profile of 2e-ORR pathway at $U=$ 
$\mathrm{OOH}^{*}$ intermediate on Edge 1A, 2A, 3A, and 4A. The EF is set at zero energy. Left side peak is anti-bonding state, right is bonding state. e, The Bader charge corresponding to S-C-N-C groups; the A sites marked with black. The blue and red region indicate electron-donating and electron-withdrawing areas, respectively.

\section{Conclusions.}

590 In summary, we have adopted SNC catalyst with S-C-N-C functional groups via 591 hydrothermal self-assembly and controllable CVD nitridation. The S-C-N-C functional 592 groups with marginal defects formed by the covalently grafting of pentagon-S and 593 pyrrolic-N onto the edge of graphene, which account for the high activity of catalyzing 594 ORHP. SNC presents excellent ORHP catalytic performance with maximum $\mathrm{H}_{2} \mathrm{O}_{2}$ yield ratio of $98.9 \%$ at $0.60 \mathrm{~V}$ vs. RHE and retains $95 \%$ of initial activity after $50000 \mathrm{~s}$ continuous reaction. DFT calculations reveal that the defective S-C-N-C functional groups in SNC afford desirable binding strength to $\mathrm{OOH}^{*}$ intermediates and diminishes 598 the energy barrier for $\mathrm{O}_{2}$ to $\mathrm{OOH}^{*}$. This work systematically elucidates the mechanism 599 of marginal defects on the performance of ORHP, which opens up an avenue for designing efficient metal-free electrocatalysts through defect engineering.

\section{Methods}

602 Preparation of SNC. GO was synthesized by using the improved Hummers method ${ }^{42}$. An aqueous suspension of GO $\left(2 \mathrm{mg} \mathrm{mL}^{-1}\right)$ was prepared by adding $0.15 \mathrm{~g}$ GO into 75 $\mathrm{mL}$ deionized water and sonicating for $10 \mathrm{~h}$. Then, $10.6875 \mathrm{mg}$ thiourea was added into the GO suspension and sonicated for another $30 \mathrm{~min}$. The obtained solution was moved 
to Teflon autoclave (100 mL volume) and heated $180{ }^{\circ} \mathrm{C}$ for $12 \mathrm{~h}$. The subsequent

607

608

609

610

611

612

613

614

615

616

617

618

619

620

621

622

623

624

625

626

627

product was freeze-dried to generate spongy column. Sponge column of S-rGO was then placed in the center of a standard 1-inch quartz tube furnace. After pumping and purging the system with $\mathrm{Ar}$ for $15 \mathrm{~min}$, the temperature was risen at $15^{\circ} \mathrm{C} \min ^{-1}$ up to $850{ }^{\circ} \mathrm{C}$ with the introducing of $\mathrm{NH}_{3}(50 \mathrm{sccm})$ and $\mathrm{Ar}(100 \mathrm{sccm})$ under a raised-up pressure ( $\sim 2.85$ Torr). The process maintained for $1 \mathrm{~h}$, and the ultimate creation SNC was cooled down to room temperature by quickly removing the sample from the hot region of furnace under the protection of Ar atmosphere.

Catalyst structural characterization. A FE-SEM (HITACHI, SU8010) and field emission gun TEM (JEOL, JEM-2100) were used to examine the morphologies and structures of the catalysts. X-ray powder diffraction (Rigaku, D/Max Ultima VI) was configured with a $\mathrm{Cu}-\mathrm{K} \alpha$ radiation source $(\lambda=1.5418 \AA)$ and graphite monochromator at $40 \mathrm{kV}$ voltage and $40 \mathrm{~mA}$ current. The Raman spectra were recorded on a confocal micro-Raman spectrometer (Horiba Jobin Yvon, LabRAM HR Evolution) with $532 \mathrm{~nm}$ laser excitation. The $\mathrm{I}_{\mathrm{D}} / \mathrm{I}_{\mathrm{G}}$ ratio of samples were calculated by deconvoluting the Raman spectra using Gaussian peak fitting (Origin 2018) to allow a comparison of peak areas. The specific area was analyzed on a Quantachrome autosorb $\mathrm{iQ}_{2}$ analyzer by $\mathrm{N}_{2}$ adsorption-desorption isotherms using the Brunauer-Emmett-Teller (BET) method. The pore size distributions were determined by the Barret-Joyner-Halenda method. Roomtemperature EPR spectra were obtained using an EMXPLUS10/12 paramagnetic resonance spectrometer, horizontal axis was convert to $g$ value by $g=h v / \beta H^{43}$. The chemical compositions of samples were confirmed by XPS spectra (ThermoFisher) 
with a monochromatic $\mathrm{Al} \mathrm{K \alpha}$ radiation. All XPS spectra were calibrated using C 1s line at $284.8 \mathrm{eV}$ and Thermo Avantage V5.52 program were employed for surface componential content analysis. Elemental content measured by elemental analyzer (Elementar, Vario EL CUBE), the oxygen content was measured separately using a linear model. FT-IR were collected on a Thermo Nicolet is 5 with a resolution of $\sim 1 \mathrm{~cm}^{-}$ ${ }^{1}$, and the samples were tableted with $\mathrm{KBr}$ as support. The XANES measurements were undertaken on the soft X-ray beamline (BL20A1 for C K- and N K- edge) and intermediate X-ray beamline (BL16A1 for S K-edge) at the National Synchrotron Radiation Research Facility at Taiwan. The sample were pressed on indium foil and measured in X-ray fluorescence mode. The data were normalized to the incident photon flux and processed using the ATHENA software package ${ }^{44}$. The Bruker AVANCE III 300 Spectrometer was conducted for Solid-state ${ }^{13} \mathrm{C}$ NMR analysis. HAADF-STEM images and EDS elemental mappings were carried out in FEI-Themis Z equipped with a Super-X EDS detector and operated at $300 \mathrm{kV}$. AC-TEM images were taken using an $80 \mathrm{KeV}$ FEI-Themis Z equipped with a spherical aberration corrector.

Electrochemical measurement. All the electrochemical measurements were performed with a three-electrode cell system operated by a $\mathrm{CHI} 760 \mathrm{E}$ ( $\mathrm{CH}$ instrument, USA) electrochemistry station and carried out in $\mathrm{O}_{2}$-saturated $0.1 \mathrm{M} \mathrm{KOH}$ electrolyte. A RRDE loaded with catalysts, a platinum wire and an $\mathrm{Ag} / \mathrm{AgCl}$ (saturated $\mathrm{KCl}$ ) were used as the working electrode, counter electrode and reference electrode, respectively. The RRDE was (AFE6R2, Pine Research Instrumentation, USA) comprised by a glassy carbon rotation disk electrode (disk area: $0.2376 \mathrm{~cm}^{2}$ ) and platinum ring (ring area: 
650

651

652

653

654

655

656

657

658

659

660

661

662

663

664

665

666

667

668

669

670

671

$0.2356 \mathrm{~cm}^{2}$ ), with a theoretical collection efficiency of 0.38 . The rotating speed of working electrode was $1600 \mathrm{rpm}$ throughout the tests. Synthetic catalyst ink was prepared by ultrasonically dispersing $2 \mathrm{mg}$ of catalyst in solution containing $40 \mu \mathrm{L}$ of 5 $\mathrm{wt} \%$ Nafion solution and $0.4 \mathrm{~mL}$ of $1: 1(\mathrm{v} / \mathrm{v})$ deionized water/ethanol. After sonication for $1 \mathrm{~h}, 5 \mu \mathrm{L}$ of the obtained suspension was loaded onto disk electrode, naturally dried prior to test. The area-based mass of loading electrocatalyst was $0.10 \mathrm{mg} \mathrm{cm}^{-2}$.

Before tests, all the electrocatalysts were activated by CV cycles with a scan rate of $100 \mathrm{mV} \mathrm{s}^{-1}$ in $\mathrm{N}_{2}$-saturated and $\mathrm{O}_{2}$-saturated $0.1 \mathrm{M} \mathrm{KOH}$ aqueous solution, the former aimed to detect background current. LSV was performed at a scan rate of $5 \mathrm{mV} \mathrm{s}^{-1}$. All the potentials were calibrated to the RHE by equation (3). The ring electrode was set as a decomposition potential of $1.2 \mathrm{~V}$ vs. RHE to assemble the $\mathrm{H}_{2} \mathrm{O}_{2}$.

$$
\mathrm{E}_{\mathrm{RHE}}=\mathrm{E}_{\mathrm{Ag} / \mathrm{AgCl}}+0.059 \times \mathrm{pH}+0.1976
$$

The selectivity of $\mathrm{H}_{2} \mathrm{O}_{2}$ and electron transfer number (n) were calculated based on the current of the disk electrode and ring electrode (equation (4) and (5)).

$$
\begin{aligned}
\mathrm{H}_{2} \mathrm{O}_{2}(\%) & =200 \times \frac{\mathrm{I}_{R} / \mathrm{N}}{\mathrm{I}_{\mathrm{D}}+\mathrm{I}_{\mathrm{R}} / \mathrm{N}} \\
\mathrm{n} & =4 \times \frac{\mathrm{I}_{\mathrm{D}}}{\mathrm{I}_{\mathrm{D}}+\mathrm{I}_{\mathrm{R}} / \mathrm{N}}
\end{aligned}
$$

where $I_{R}$ is the ring current, ID is the disk current and $\mathrm{N}$ is the collection efficiency.

The ECSA of the electrode was determined by measuring the $\mathrm{C}_{\mathrm{dl}}$ of the catalytic surface, which is derived from the CV cycles (Scan rates were 20, 60, 100, 140, 180, 220,260 and $300 \mathrm{mV} \mathrm{s}^{-1}$ ) measured at the range of 0.86 to $1.06 \mathrm{~V}$ vs. RHE where no Faradaic current was generated. Based on the definition: ECSA $=\mathrm{C}_{\mathrm{dl}} / \mathrm{C}_{\mathrm{s}}$, we used a specific capacitance (Cs) value of $40 \mu \mathrm{F} \mathrm{cm} \mathrm{cm}^{-2}$.

Computational details. All the calculations were performed using Vienna ab initio 
672 Simulation Package (VASP 6.2) with the projector augmented wave (PAW) 673 pseudopotential method ${ }^{45}$. The exchange-correlation potential was described by 674 Perdew-Burke-Ernzerhof (PBE) functional ${ }^{46}$ with generalized gradient approximation 675 (GGA). The cutoff energy for the plane-wave was set to $500 \mathrm{eV}$. In the geometry 676 optimization, the convergence criteria for the energy and force were $10^{-4} \mathrm{eV}$ and $10^{-2}$ $677 \mathrm{eV} \AA^{-1}$, respectively. A large vacuum spacing (more than $15 \AA$ ) was taken to prevent 678 mirror interactions. The Brillouin zones were sampled with $2 \pi \times 0.02 \AA^{-1}$ spacing in 679 reciprocal space by the Monkhorst-Pack scheme. Grimme's DFT-D3 van der Waals 680 (vdW) corrections with the Becke-Jonson (BJ) damping was employed. The Gibbs 681 reaction-free energy change $(\Delta \mathrm{G})$ for each step in the ORR was calculated using the 682 computational hydrogen electrode (CHE) $\operatorname{model}^{47}$,

$$
\Delta \mathrm{G}=\Delta \mathrm{E}+\Delta \mathrm{ZPE}-\mathrm{T} \Delta \mathrm{S}+\mathrm{eU}+\Delta \mathrm{G}_{\mathrm{pH}}
$$

684

where $\Delta \mathrm{E}, \Delta \mathrm{ZPE}$ and $\Delta \mathrm{S}$ are the binding energy change, zero-point energy change and entropy change between the reactant and product. $U$ and e are the electrode potential and the number of electrons transferred, respectively. $\Delta \mathrm{G}_{\mathrm{pH}}$ is the free energy correction of $\mathrm{pH}$ calculated by equation (7):

$$
\Delta \mathrm{G}_{\mathrm{pH}}=\ln 10 \times \mathrm{k}_{\mathrm{B}} \mathrm{T} \times \mathrm{pH}
$$

Since RHE is taken as the reference, the $\mathrm{pH}$ was set to be zero in this calculation. The $\mathrm{UL}_{\mathrm{L}}$ is defined as the lowest potential at which all the reaction steps are downhill in free energy.

Data availability. The data that support the plots within these paper and other findings of this study are available from the corresponding author upon reasonable request. 


\section{References}

1. Campos-Martin, J. M., Blanco-Brieva, G. \& Fierro, J. L. G. Hydrogen peroxide synthesis: an outlook beyond the anthraquinone process. Angew. Chem. Int. Ed. 45, 6962-6984 (2006).

2. Kiran Pulidindi \& Hemant Pandey. Hydrogen peroxide market by end-user (paper \& pulp, chemical, waste water treatment, mining), industry analysis report, regional outlook, application potential, price trends, competitive market share \& forecast, 2020-2026.

https://www.gminsights.com/pressrelease/hydrogen-peroxide-market.

3. Siahrostami, S. et al. Enabling direct $\mathrm{H}_{2} \mathrm{O}_{2}$ production through rational electrocatalyst design. Nat. Mater. 12, 1137-1143 (2013).

4. Jung, E. et al. Atomic-level tuning of Co-N-C catalyst for high-performance electrochemical $\mathrm{H}_{2} \mathrm{O}_{2}$ production. Nat. Mater. 19, 436-442 (2020).

5. Verdaguer-Casadevall, A. et al. Trends in the electrochemical synthesis of $\mathrm{H}_{2} \mathrm{O}_{2}$ : enhancing activity and selectivity by electrocatalytic site engineering. Nano Lett. 14, 1603-1608 (2014).

6. Freakley, S. J. et al. Palladium-tin catalysts for the direct synthesis of $\mathrm{H}_{2} \mathrm{O}_{2}$ with high selectivity. Science. 351, 965-968 (2016).

7. Wang, Z. et al. Hydrogen peroxide generation with $100 \%$ faradaic efficiency on metal-free carbon black. ACS Catal. 11, 2454-2459 (2021).

8. Lu, Z. et al. High-efficiency oxygen reduction to hydrogen peroxide catalysed by oxidized carbon materials. Nat. Catal. 1, 156-162 (2018). 
9. Han, G. F. et al. Building and identifying highly active oxygenated groups in carbon materials for oxygen reduction to $\mathrm{H}_{2} \mathrm{O}_{2}$. Nat. Commun. 11, 2209 (2020).

10. Xia, C., Xia, Y., Zhu, P., Fan, L. \& Wang, H. Direct electrosynthesis of pure aqueous $\mathrm{H}_{2} \mathrm{O}_{2}$ solutions up to $20 \%$ by weight using a solid electrolyte. Science. 366, 226-231 (2019).

11. Perazzolo, V. et al. Nitrogen and sulfur doped mesoporous carbon as metal-free electrocatalysts for the in situ production of hydrogen peroxide. Carbon. 95, 949-963 (2015).

12. Yu, D., Zhang, Q. \& Dai, L. Highly efficient metal-free growth of nitrogendoped single-walled carbon nanotubes on plasma-etched substrates for oxygen reduction. J. Am. Chem. Soc. 132, 15127-15129 (2010).

13. Tang, C. \& Zhang, Q. Nanocarbon for oxygen reduction electrocatalysis: dopants, edges, and defects. Adv. Mater. 29, 1604103 (2017).

14. De Andres, P. L., Ramírez, R. \& Vergés, J. A. Strong covalent bonding between two graphene layers. Phys. Rev. B 77, 1-5 (2008).

15. Stobinski, L. et al. Graphene oxide and reduced graphene oxide studied by the XRD, TEM and electron spectroscopy methods. J. Electron Spectros. Relat. Phenomena 195, 145-154 (2014).

16. Stanford, M. G. et al. Flash graphene morphologies. ACS Nano 14, 13691$13699(2020)$.

17. Lv, C. et al. Defect engineering metal-free polymeric carbon nitride electrocatalyst for effective nitrogen fixation under ambient conditions. Angew. 
Chem. Int. Ed. 130, 10403-10407 (2018).

18. Ardelean, I., Cozar, O., Filip, S., Pop, V. \& Cenan, I. EPR and magnetic susceptibility studies of $\mathrm{Cu}^{2+}$ ions in $\mathrm{Bi}_{2} \mathrm{O}_{3} \cdot \mathrm{GeO}_{2}$ glasses. Solid State Commun. 100, 609-613 (1996).

19. Yang, S. et al. Efficient synthesis of heteroatom ( $\mathrm{N}$ or S)-doped graphene based on ultrathin graphene oxide-porous silica sheets for oxygen reduction reactions. Adv. Funct. Mater. 22, 3634-3640 (2012).

20. Wu, K.-H. et al. Highly selective hydrogen peroxide electrosynthesis on carbon: in situ interface engineering with surfactants. Chem 6, 1443-1458 (2020).

21. Bagri, A. et al. Structural evolution during the reduction of chemically derived graphene oxide. Nat. Chem. 2, 581-587 (2010).

22. Li, D. et al. A defect-driven metal-free electrocatalyst for oxygen reduction in acidic electrolyte. Chem 4, 2345-2356 (2018).

23. Lindberg, B. J. et al. Molecular spectroscopy by means of ESCA II. sulfur compounds. Correlation of electron binding energy with structure . Phys. Scr. 1, 286-298 (1970).

24. Zhang, B., Zhou, Y., Zhang, J. \& Wang, Z. The effect of thermal annealing on the microstructure and mechanical properties of magnetron sputtered hydrogenated amorphous carbon films. Surf. Interface Anal. 44, 162-165 (2012).

25. Ray, S. C. et al. Electronic properties of a-CN $\mathrm{N}_{\mathrm{x}}$ thin films: an $\mathrm{x}$-ray-absorption 
and photoemission spectroscopy study. J. Appl. Phys. 98, 33708 (2005).

761

762

26. Pacilé, D. et al. Near-edge X-ray absorption fine-structure investigation of graphene. Phys. Rev. Lett. 101, 1-4 (2008).

27. Zhu, Q., Money, S. L., Russell, A. E. \& Thomas, K. M. Determination of the fate of nitrogen functionality in carbonaceous materials during pyrolysis and combustion using X-ray absorption near edge structure spectroscopy. Langmuir 13, 2149-2157 (1997).

28. Zhang, L. S., Liang, X. Q., Song, W. G. \& Wu, Z. Y. Identification of the nitrogen species on $\mathrm{N}$-doped graphene layers and $\mathrm{Pt} / \mathrm{NG}$ composite catalyst for direct methanol fuel cell. Phys. Chem. Chem. Phys. 12, 12055-12059 (2010).

29. Li, L. et al. Tailoring selectivity of electrochemical hydrogen peroxide generation by tunable pyrrolic-nitrogen-carbon. Adv. Energy Mater. 10, 1-10 (2020).

30. Prietzel, J., Thieme, J., Neuhäusler, U., Susini, J. \& Kögel-Knabner, I. Speciation of sulphur in soils and soil particles by X-ray spectromicroscopy. Eur. J. Soil Sci. 54, 423-433 (2003).

31. Sathe, B. R., Zou, X. \& Asefa, T. Metal-free B-doped graphene with efficient electrocatalytic activity for hydrogen evolution reaction. Catal. Sci. Technol. 4, 2023-2030 (2014).

32. Prietzel, J. et al. Sulfur speciation in soil by S K-edge XANES spectroscopy: comparison of spectral deconvolution and linear combination fitting. Environ. Sci. Technol. 45, 2878-2886 (2011). 
33. Weiwei, C. et al. Synthesis and solid-state NMR structural characterization of ${ }^{13}$ C-Labeled graphite oxide. Science. 321, 1815-1817 (2008).

34. Yan, Z. et al. Hexagonal graphene onion rings. J. Am. Chem. Soc. 135, 10755$10762(2013)$.

35. Banhart, F., Kotakoski, J. \& Krasheninnikov, A. V. Structural defects in graphene. ACS Nano 5, 26-41 (2011).

36. Stone, A. J. \& Wales, D. J. Theoretical studies of icosahedral C60 and some related species. Chem. Phys. Lett. 128, 501-503 (1986).

37. Sa, Y. J., Kim, J. H. \& Joo, S. H. Active edge-site-rich carbon nanocatalysts with enhanced electron transfer for efficient electrochemical hydrogen peroxide production. Angew. Chem. Int. Ed. 58, 1100-1105 (2019).

38. Peng, X. \& Ahuja, R. Symmetry breaking induced bandgap in epitaxial graphene layers on SiC. Nano Lett. 8, 4464-4468 (2008).

39. Perry, S. C. et al. Electrochemical synthesis of hydrogen peroxide from water and oxygen. Nat. Rev. Chem. 3, 442-458 (2019).

40. Guo, D. et al. Active sites of nitrogen-doped carbon materials for oxygen reduction reaction clarified using model catalysts. Science. 351, 361-365 (2016).

41. Yang, Q. et al. Atomically dispersed Lewis acid sites boost 2-electron oxygen reduction activity of carbon-based catalysts. Nat. Commun. 11, 5478 (2020).

42. Marcano, D. C. et al. Improved synthesis of graphene oxide. ACS Nano 4, 4806-4814 (2010). 
43. Yen, A. S., Kim, S. S., Hecht, M. H., Frant, M. S. \& Murray, B. Evidence that the reactivity of the martian soil is due to superoxide ions. Science. 289, 19091912 (2000).

44. Ravel, B. \& Newville, M. ATHENA, ARTEMIS, HEPHAESTUS: data analysis for X-ray absorption spectroscopy using IFEFFIT. J. Synchrotron Radiat. 12, 537-541 (2005).

45. Joubert, D. From ultrasoft pseudopotentials to the projector augmented-wave method. Phys. Rev. B 59, 1758-1775 (1999).

46. Perdew, J. P., Burke, K. \& Ernzerhof, M. Generalized gradient approximation made simple. Phys. Rev. Lett. 77, 3865-3868 (1996).

47. Nørskov, J. K. et al. Origin of the overpotential for oxygen reduction at a fuelcell cathode. J. Phys. Chem. B 108, 17886-17892 (2004).

\section{Acknowledgments}

The authors acknowledge National Natural Science Foundation of China (No. 22175109), Natural Science Foundation of Excellent Young Scholars for Shanxi Province (201901D211198), and the Program for the Outstanding Innovative Teams of Higher Learning Institutions of Shanxi (OIT, No. 231544901002) for finance support of this research. L. L. acknowledges the support from the Discovery Program by the Natural Sciences and Engineering Research Council Canada (NSERC, DG RGPIN2020-06675). We also thank Dr. Bo Chen from Rice University for assistance with XPS spectroscopy. The authors acknowledge Prof. Chuan Xia at University of Electronic Science and Technology of China for his valuable scientific discussion. The authors 
826 would also like to acknowledge Dr. Junjie Zhang from Scientific Instrument Center at

827 Shanxi University for her help with ICP-MS measurement.

828 Author contributions

829 Z. M., Y. M., Y. Q., and X. F. conceived the experiment and scientific discussions. Z.

830 M. carried out the syntheses and electrocatalysis measurements. D. C., L. L., S. C., W.

831 Y., T. C., L. C., and H. Z. carried out the characterizations. Z. M. and X. F. wrote the 832 paper. All the authors discussed the results and revised the paper.

\section{Competing interests}

834 The authors declare no competing financial interests.

835 Additional information

836 Supplementary information is available in the online version of the paper.

837 Reprints and permission information is available at www.nature.com/reprints.

838 Correspondence and requests for materials should be addressed to Y. Q. or X. F. 


\section{Supplementary Files}

This is a list of supplementary files associated with this preprint. Click to download.

- SupportInformation.pdf 